\title{
Diffusion of rod-like nanoparticles in non-adhesive and adhesive porous polymeric gels
}

\author{
Jiuling Wang ${ }^{\mathrm{a}, c, \mathrm{~d}, 1}$, Yiwei Yang ${ }^{\mathrm{b}, \mathrm{d}, 1}$, Miaorong Yu ${ }^{\mathrm{b}, \mathrm{d}}$, Guoqing $\mathrm{Hu}^{\mathrm{c}, \mathrm{d}}$, Yong Gan ${ }^{\mathrm{b}, \mathrm{d}, *}$, \\ Huajian Gao ${ }^{\mathrm{e}, * *}$, Xinghua Shi ${ }^{\mathrm{a}, \mathrm{c}, \mathrm{d}, * * * *}$ \\ ${ }^{a}$ CAS Key Laboratory for Nanosystem and Hierarchy Fabrication, CAS Center for Excellence in Nanoscience, National Center for \\ Nanoscience and Technology, Chinese Academy of Sciences, Beijing 100190, China \\ ${ }^{\mathrm{b}}$ Shanghai Institute of Materia Medica, Chinese Academy of Sciences, Shanghai 201203, China \\ ${ }^{\mathrm{c}}$ LNM, Institute of Mechanics, Chinese Academy of Sciences, Beijing 100190, China \\ ${ }^{\mathrm{d}}$ University of Chinese Academy of Sciences, NO.19A Yuquan Road, Beijing 100049, China \\ e School of Engineering, Brown University, Providence, RI 02912, USA
}

\section{A R T I C L E I N F O}

\section{Article history:}

Received 3 December 2017

Accepted 30 December 2017

Available online 3 January 2018

\section{Keywords:}

Nanoparticle diffusion

Shape effect

Mucus

Nanorods

Porous media

Mean first passage time

\begin{abstract}
A B S T R A C T
It is known that rod-like nanoparticles (NPs) can achieve higher diffusivity than their spherical counterparts in biological porous media such as mucus and tumor interstitial matrix, but the underlying mechanisms still remain elusive. Here, we present a joint experimental and theoretical study to show that the aspect ratio (AR) of NPs and their adhesive interactions with the host medium play key roles in such anomalous diffusion behaviors of nanorods. In an adhesive polymer solution/gel (e.g., mucus), hopping diffusion enables nanorods to achieve higher diffusivity than spherical NPs with diameters equal to the minor axis of the rods, and there exists an optimal AR that leads to maximum diffusivity. In contrast, the diffusivity of nanorods decreases monotonically with increasing AR in a nonadhesive polymer solution/gel (e.g., hydroxyethyl cellulose, HEC). Our theoretical model, which captures all the experimental observations, generalizes the so-called obstructionscaling model by incorporating the effects of the NPs/matrix interaction via the mean first passage time (MFPT) theory. This work reveals the physical origin of the anomalous diffusion behaviors of rod-like NPs in biological gels and may provide guidelines for a range of applications that involve NPs diffusion in complex porous media.
\end{abstract}

(c) 2018 Elsevier Ltd. All rights reserved.

\section{Introduction}

Increasing the transport rate of NPs through physiological barriers, prominent examples including the gel-like mesh structures of mucosa and tumor interstitial matrix, is crucial for improving the efficiency of drug delivery systems (Jain and Stylianopoulos, 2010; Lai et al., 2009). Mucus, a tenacious gel that protects mucosal surfaces from the entry of external pathogens (Button et al., 2012; Johansson et al., 2013; McGuckin et al., 2011), controls the entry of drug carriers into the

\footnotetext{
* Corresponding author at: Shanghai Institute of Materia Medica, Chinese Academy of Sciences, Shanghai 201203, China.

** Corresponding author.

*** Corresponding author at: National Center for Nanoscience and Technology, Chinese Academy of Sciences, 11 Beiyitiao Road, Zhongguancun, Beijing 100190, China.

E-mail addresses: ygan@simm.ac.cn (Y. Gan), huajian_Gao@brown.edu (H. Gao), shixh@nanoctr.cn (X. Shi).

1 These authors contributed equally to this work.
} 
Table 1

Parameters of nanoparticles (MSNs) used in the experiments.

\begin{tabular}{llllll}
\hline Nanoparticle & Aspect ratio & Size in SEM $(\mathrm{nm})$ & Hydrodynamic diameter $(\mathrm{nm})$ & PDI & Zeta potential $(\mathrm{mV})$ \\
\hline AR1 & 1.0 & 80 & $108 \pm 12$ & 0.022 & $-3.6 \pm 1.0$ \\
AR2 & 1.9 & $75 \times 145$ & $160 \pm 18$ & 0.014 & $-4.7 \pm 1.2$ \\
AR3 & 2.8 & $78 \times 220$ & $210 \pm 20$ & 0.060 & $-2.1 \pm 0.7$ \\
AR4 & 4.1 & $85 \times 350$ & $257 \pm 33$ & 0.089 & $-1.0 \pm 0.7$ \\
AR8 & 7.7 & $65 \times 500$ & $302 \pm 48$ & 0.077 & $-2.6 \pm 0.9$ \\
\hline
\end{tabular}

circulatory system (Cone, 2009; Lai et al., 2009). The tumor interstitial matrix, another polymer gel-like medium, constitutes a vital barrier to the delivery of drug-loaded NPs to tumor cells (Jain and Stylianopoulos, 2010). Xue et al. developed a biochemomechanical model for the growth of tumor interstitial matrix (Xue et al., 2016) and a non-equilibrium thermodynamics model to capture the effect of tumor matrix on drug transport (Xue et al., 2017). In addition to their steric hindrance effect on the movement of NPs (Hofling and Franosch, 2013; Saxton, 1994; Stylianopoulos et al., 2010a), fibrous media also exhibit heterogeneous interactions with NPs, including non-specific interactions (van der Waals, electrostatic and hydrophobic interactions) and specific interactions between complementary structures (Lai et al., 2009; Ponchel and Irache, 1998; Saxton, 1996; Stylianopoulos et al., 2010b), which can reduce the mobility of NPs by orders of magnitude. These fundamental characteristics of biological media suggest possibilities for rational and mechanism-based design of drug delivery systems. The shape of NPs is known to strongly affect their intravenous circulation time (Geng et al., 2007), translocation across cell membrane (Li et al., 2013; Shi et al., 2011; Wang et al., 2014; Yang and Ma, 2010), and intracellular transport route (Hinde et al., 2017). So far, relatively few studies have focused on the effect of the shape of NPs on their transport in porous media (Choi et al., 2015; Fakhri et al., 2010; Han et al., 2006; Lee et al., 2017; Peng et al., 2016). Recently, it has been shown that the diffusivity in mucus of nanorods with diameter $\times$ length of $80 \times 240 \mathrm{~nm}$ is more than 3 times higher than that of nanospheres with diameter of $80 \mathrm{~nm}$ (Yu et al., 2016). Another study reported that nanorods penetrate more efficiently through the tumor interstitial matrix than nanospheres of the same hydrodynamic radius (Chauhan et al., 2011). These anomalous diffusion behaviors associated with particle shape could open a new route to more efficient NP-based drug delivery systems, and this calls for a systematic investigation of the underlying physical mechanisms.

Various diffusion models based on obstruction effects (Johansson et al., 1991; Tomadakis and Sotirchos, 1993), hydrodynamic interactions (Altenberger et al., 1986; Cukier, 1984), free volume theory (Yasuda et al., 1968), polymer scaling theory (Cai et al., 2011, 2015) and a microscopic statistical mechanical theory (Zhang and Schweizer, 2016) have been developed to describe the diffusion of NPs in polymer solutions and gels (Masaro and Zhu, 1999). In particular, an obstruction-scaling model was developed for the diffusion of spherical solutes in covalently cross-linked hydrogels (Amsden, 1998, 1999; Hadjiev and Amsden, 2015), and later used to describe the diffusion of spherical NPs in mucus (Cu and Saltzman, 2009; Olmsted et al., 2001) and to estimate the pore size of human cervicovaginal mucus ( $340 \mathrm{~nm}$ ) (Lai et al., 2010). However, this model is not applicable to the diffusion of NPs in adhesive mucus, as it neglects adhesive interactions between solute particles and matrix. In reality, diffusing NPs can strongly interact with mucus, where the diffusivity of uncoated NPs can be three orders of magnitude lower than that of polyethylene glycol (PEG)-coated NPs (Lai et al., 2007). Therefore, it is imperative to develop a theoretical model that accounts for the interactions between NPs and matrix.

This paper is aimed to present a joint experimental and theoretical study to elucidate how the shape of NPs affects their diffusion in non-adhesive and adhesive polymeric gels. In Section 2, we report experimentally measured diffusivity of NPs with different aspect ratios in mucus and hydroxyethyl cellulose (HEC). In Section 3, we extend Amsden's obstruction-scaling model to describe the diffusion of nanorods in non-adhesive polymer solutions/gels. In Section 4, we establish a framework based on the mean first passage time (MFPT) theory to incorporate the effect of NP/polymer affinity and study how the aspect ratio affects the diffusivity of NPs. Our theoretical model pinpoints the underlying mechanisms behind the shape effect in the diffusion of NPs in non-adhesive and adhesive porous gels, and explains all experimental observations. Finally, Section 5 summarizes the main conclusions of this study.

\section{Experimental observations}

Mucus was used as a model system to investigate how the shape of NPs affects their diffusion in an adhesive porous medium, where the mucin fibers exhibit alternating hydrophilic and hydrophobic regions. The hydrophobic regions are folded and cross-linked to form hydrophobic globules (Cone, 2009) within which even PEG-coated NPs can be trapped (Lai et al., 2007; Wang et al., 2008; Xu et al., 2015). By adjusting the concentration of reagents and temperature, we fabricated mesoporous silica NPs (MSNs) with the same minor-axis diameter but different aspect ratios: $A R=1,2,3,4$, and 8, denoted as AR1, AR2, AR3, AR4 and AR8, respectively (Fig. 1). These NPs have nearly neutral zeta potentials to ensure that the AR is the sole variable and to avoid the influence of surface charge on the NP-mucin interactions (Table 1). The diameter of the NPs was controlled to vary in a narrow range. Rhodamine isothiocyanate (RITC) was labeled onto the surface of MSNs by grafting of RITC-APTES ((3-Aminopropyl)triethoxysilane) (Yu et al., 2016). More details about the experiments are provided in Appendix B. Multiple particle tracking techniques were used to monitor the movement trajectory, mean square displacement (MSD) and diffusivity of NPs in rat intestinal mucus (Fig. 2). We analyzed 300 particles from each group. The accumulative 

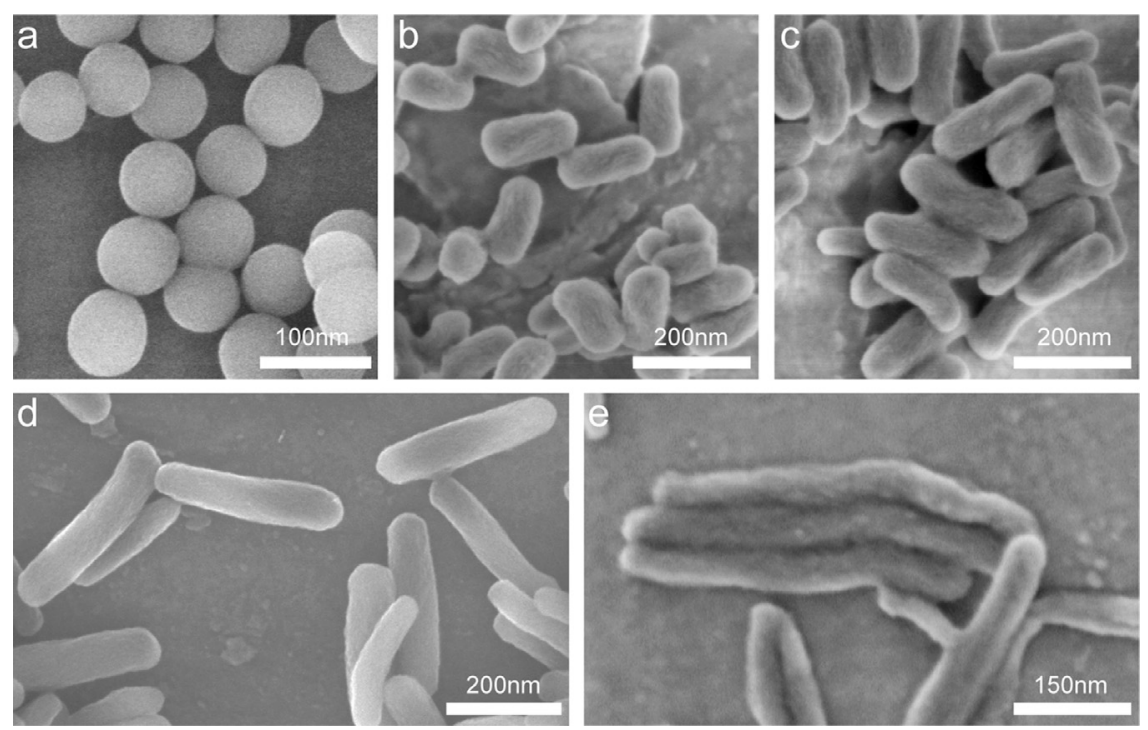

Fig. 1. Scanning electron microscopic (SEM) images of MSNs with different ARs. (a) AR1 with diameter around $80 \mathrm{~nm}$. (b) AR2 with diameter $\times$ length of $75 \times 145 \mathrm{~nm}$. (c) AR3 with diameter $\times$ length of $78 \times 220 \mathrm{~nm}$. (d) AR4 with diameter $\times$ length of $85 \times 350 \mathrm{~nm}$. (e) AR8 with diameter $\times$ length of $65 \times 500 \mathrm{~nm}$.

Table 2

Typical parameters for mucus system and NPs.

\begin{tabular}{lll}
\hline Parameters & Value & Explanation \\
\hline $2 v L$ & $16 \times 10^{13} / \mathrm{m}^{2}$ & Total fiber length per unit volume \\
$2 L$ & $1 \mu \mathrm{m}$ & Length of the fibers \\
$R$ & $40 \mathrm{~nm}, 50 \mathrm{~nm}, 60 \mathrm{~nm}$ & Radius of NPs \\
$R_{H}$ & $70 \mathrm{~nm}, 80 \mathrm{~nm}, 90 \mathrm{~nm}, 100 \mathrm{~nm}$ & Hydrodynamic radius of NPs \\
\hline
\end{tabular}

MSDs at $1 \mathrm{~s}$ were $0.29,0.74,0.87,0.34$ and $0.014 \mu \mathrm{m}^{2}$ for MSNs with AR of $1,2,3,4$ and 8 , respectively (Fig. 2(b)). These results indicate that the diffusivities of AR2 and AR3 are significantly higher than that of AR1 (i.e. spherical NPs). Furthermore, a rapid decrease in diffusivity was observed when the AR increases to 4 and 8 , suggesting the existence of an optimal $\operatorname{AR}(\sim 3)$ for the maximum transport rate in adhesive mucus.

To test whether the high affinity between NPs and mucin fibers is a key factor that leads to the observed anomalous diffusion behaviors, we also investigated the diffusivity of MSNs in hydroxyethyl cellulose (HEC, 0.5\%, w/w), a hydrophilic and non-ionic gel without hydrophobic regions (Vadodaria and English, 2016). HEC has been used to mimic the mesh structure of mucus, and its porous structure has also been confirmed via cryogenic scanning EM (cryo-SEM) (Kirch et al., 2012). Here HEC powder was dissolved in deionized water with a concentration of $0.5 \%(w / w)$. As shown in Fig. 2, the diffusivities of MSNs monotonically decline with increasing AR. Thus, unlike mucus with hydrophobic and adhesive cores at the intersection of mucin fibers, the HEC fibers could not exert a strong interaction on the NPs. This is consistent with our intuition that in the absence of sufficiently strong adhesion, increased AR retards the diffusion of MSNs.

We further investigated the mobility of MSNs in glycerine $(80 \%, \mathrm{v} / \mathrm{v})$, a solution with no porous network but high viscosity. Again, the diffusivities of MSNs monotonically decline with increasing AR (Fig. 2). These results indicate that the adhesion regions in the porous network of mucus play an essential role in the enhanced diffusivity of nanorods.

\section{Non-adhesive diffusion model}

We first extend Amsden's obstruction-scaling model to describe the diffusion of nanorods in non-adhesive porous media (Fig. 3(a)). In this model, the diffusivity of spherical NPs in polymer gels is described through the probability of one NP encountering a series of openings between the polymer chains whose spacing is larger than the size of the NP (Amsden, 1999). For spherical NPs, the normalized diffusivity is

$$
\kappa=\frac{D_{g}}{D_{0}}=\int_{R}^{\infty} g(r) d r=P(r>R),
$$

where $D_{g}$ is the diffusivity of the NPs in the polymer solution; $D_{0}$ is the diffusivity of the NPs in water; $\kappa$ is the ratio between $D_{g}$ and $D_{0} ; g(r)$ is the distribution function of the radii $r$ of the openings between polymer chains; and $R$ is the radius of the spherical NPs. 


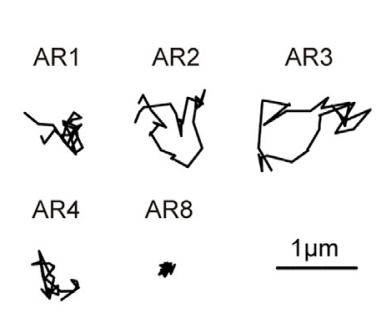

d

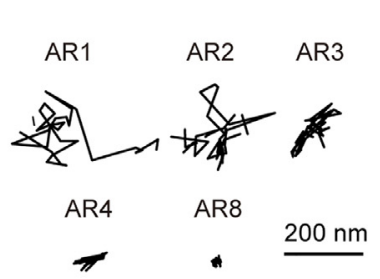

9

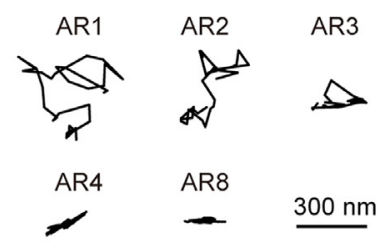

b

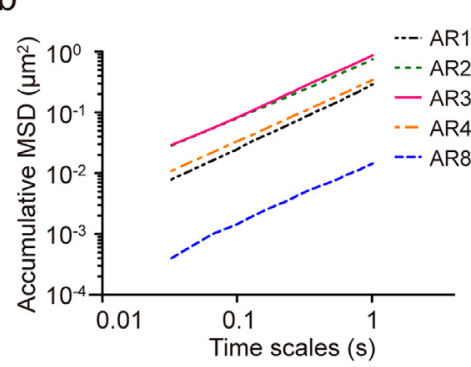

e

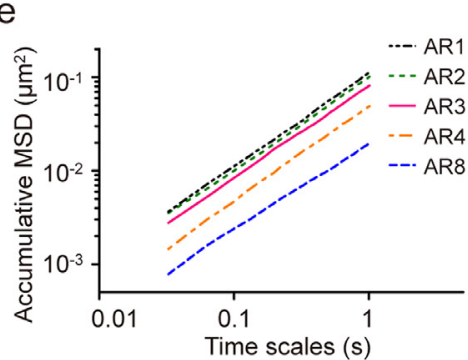

$\mathrm{h}$

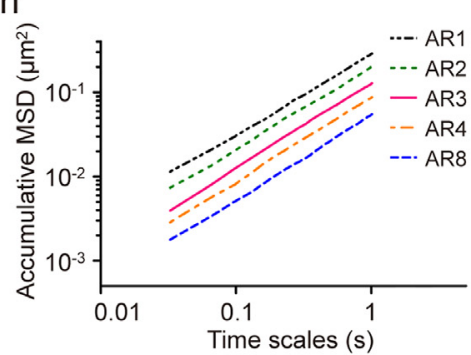

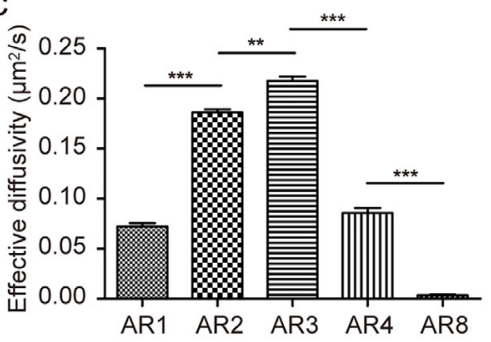

$f$

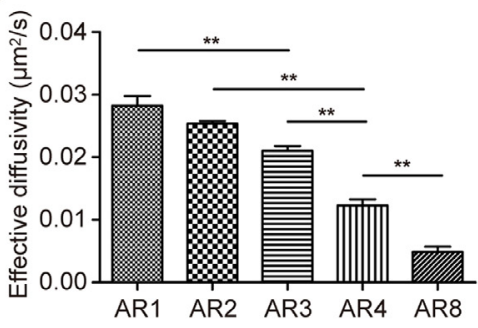

i

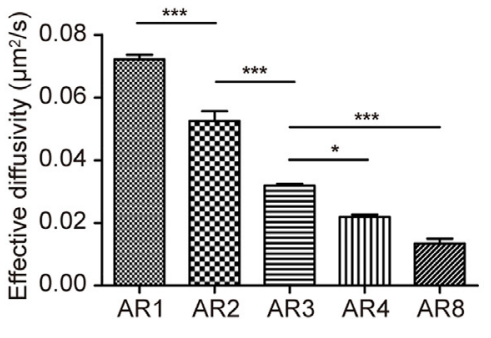

Fig. 2. Multiple particle tracking of MSNs in mucus (a), (b) and (c), $0.5 \%$ HEC (d), (e) and (f) and $80 \%$ glycerine (g), (h) and (i) at room temperature. (a), (d) and $(\mathrm{g})$, Representative trajectories of MSNs with different ARs over a time period of $1 \mathrm{~s}$. (b), (e) and (h), Ensemble-averaged accumulative MSD of MSNs diffusing over a time period of $1 \mathrm{~s}$. (c), (f) and (i), Effective diffusivities $\left(D_{\text {eff }}\right)$ of NPs on a time scale of $1 \mathrm{~s}$. More than 300 particles were analyzed for each sample. ${ }^{* *} P<0.01,{ }^{* * *} P<0.001$, Bonferroni's test. Data are means \pm SEM.

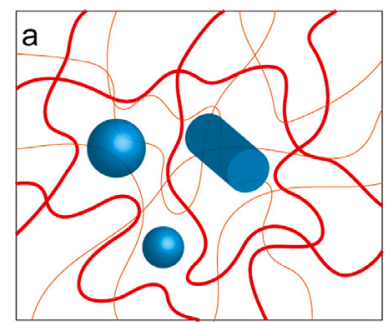

C

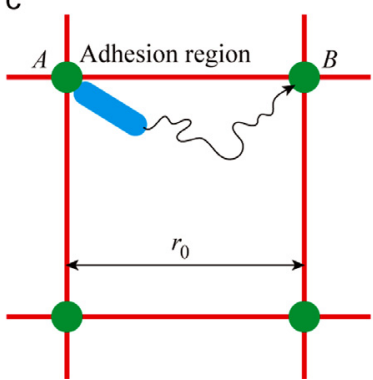

b

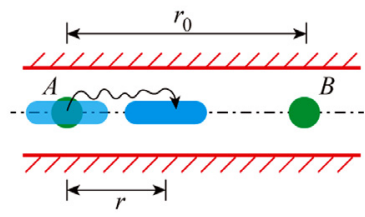

d

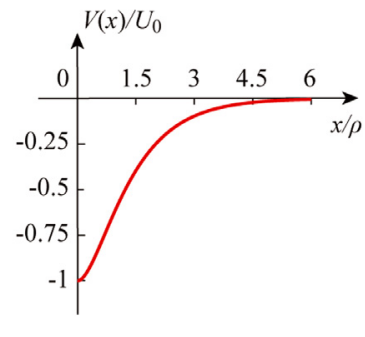

Fig. 3. Diffusion of NPs in a polymer gel. (a) Schematic showing diffusion of spherical and rod-like NPs in a polymer gel. (b) Schematic showing NP diffusion in one-dimensional (1D) cylindrical nanopores with adhesion regions A and B. (c) Schematic showing NP diffusion in a three-dimensional (3D) regular network with adhesion regions. (d) Morse potential $V(x)=U_{0}\left(e^{-2 x / \rho}-2 e^{-x / \rho}\right)$ is used to describe the interaction energy between nanorods per unit length and the adhesion region, where $U_{0}$ is the adhesion strength, $x$ is the distance between the NP and the adhesion region, and $\rho$ is the potential range. 

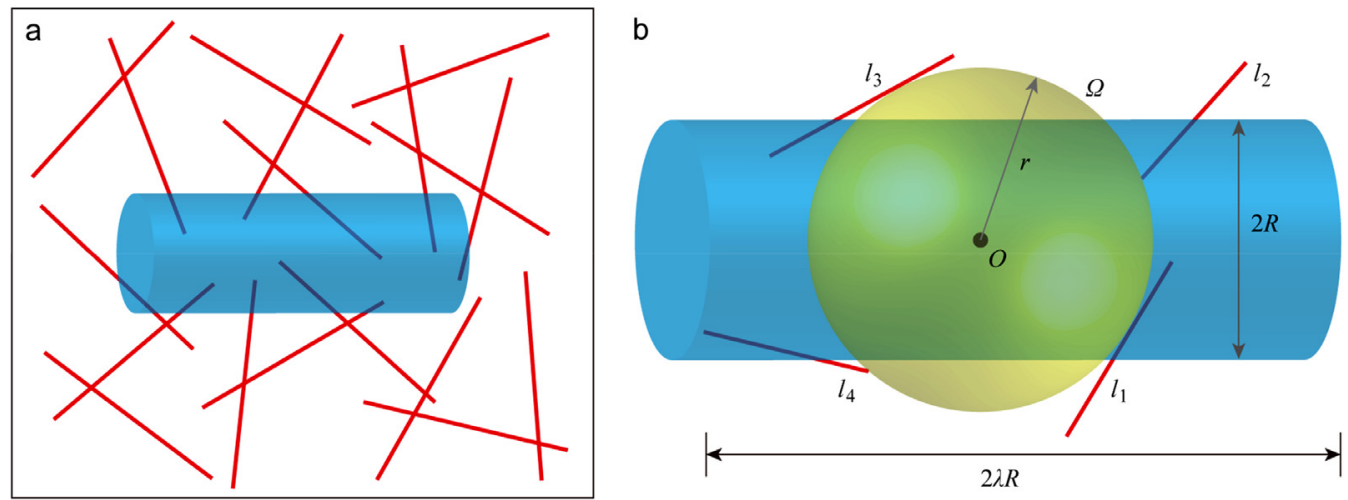

Fig. 4. Contact between fibers and a rod NP. (a) Schematic showing a cylinder in a random network composed of straight fibers. (b) Different contact manners between NPs and spherical surface $\boldsymbol{\Omega}$ when NPs are placed in the random network of straight fibers. $\boldsymbol{\Omega}$ is a spherical surface expanded from point $O$ until it reaches the fibers, and $r$ is the radius of $\boldsymbol{\Omega}$ and referred to as the contact distance.

The hydrodynamic radius of the rod-like NPs, $R_{H}$, is evaluated as (Ortega and de la Torre, 2003)

$$
R_{H}=\left(\frac{3}{2} \lambda\right)^{1 / 3} R\left[1.009+1.395 \times 10^{-2} \ln \lambda+7.88 \times 10^{-2}(\ln \lambda)^{2}+6.04 \times 10^{-3}(\ln \lambda)^{3}\right],
$$

where $\lambda$ is the AR of the cylinder within $0.1<\lambda<30$. The diffusion coefficient of NPs in water, $D_{0}$, can be evaluated by the Stokes-Einstein equation

$$
D_{0}=\frac{k_{B} T}{6 \pi \eta \cdot R_{H}},
$$

where $\eta$ is the dynamic viscosity of water, $k_{B}$ is the Boltzmann constant, and $T$ is temperature. Combining Eqs. (2) and (3), one can estimate the diffusion coefficient of rod-like NPs.

Ogston showed that the radii of spherical spaces between a random network composed of straight fibers of negligible width obey the following distribution (Ogston, 1958),

$$
g(r)=\left(4 \pi v L r+4 \pi v r^{2}\right) \cdot \exp \left[-\left(2 \pi v L r^{2}+\frac{4 \pi}{3} v r^{3}\right)\right],
$$

where $v$ is the average number of fibers per unit volume and $L$ is the half length of the fibers. Using the distribution of openings in such a random network to approximate the openings between the polymer chains (Hadjiev and Amsden, 2015), Amsden obtained the normalized diffusivity of spherical NPs. In fact, mathematically, Eq. (1) is equivalent to the probability that there are no fibers in contact with the spherical NP when the NP is placed in the random network. Following this framework, consideration of a random network composed of straight fibers leads to the average number of fibers in contact with a rod-like NP, $\bar{N}$, while the actual number $N$ of fibers in contact with the rod-like NP follows a Poisson distribution (Chun et al., 1969; Rodbard and Chrambac, 1970)

$$
P(N=k)=\frac{\bar{N}^{k} \cdot \exp (-\bar{N})}{k !} .
$$

Eq. (5) describes the fraction of space that is penetrated by $k$ fibers; when $k=0$, it is the fraction of space that can accommodate the NPs. The model is based on the following assumptions: 1) The relaxation of polymer chains is much slower than the diffusion of NPs so that the polymer matrix can be considered immobile; 2 ) The distribution of the openings between polymer chains can be approximated by that in a random network composed of straight fibers. Accordingly, the normalized diffusivity of the nanorods, i.e. the probability of a rod-like NP encountering a series of openings between the polymer chains whose spacing is larger than its size, is expressed as

$$
\kappa=\frac{D_{g}}{D_{0}}=P(N=0)=\exp (-\bar{N}) .
$$

\subsection{Average number of fibers in contact with rod-like NPs}

Next, we determine the average number of fibers in contact with randomly dispersed rod-like NPs in a fiber network. As shown in Fig. 4, $O$ is the center of a NP, $\boldsymbol{\Omega}$ is a spherical surface expanded from point $O$ until it reaches the fibers, and $r$ is the radius of $\boldsymbol{\Omega}$ and contact distance. Depending on the contact manner between the NP and $\boldsymbol{\Omega}$, the fibers can be classified into four kinds: (1) in tangential contact with $\Omega$ and the contact points are within the NP $\left(l_{1}\right)$; (2) end-point contact and the contact points are within the NP $\left(l_{2}\right)$; (3) tangential contact and the contact points are outside the NP $\left(l_{3}\right)$; (4) end-point 

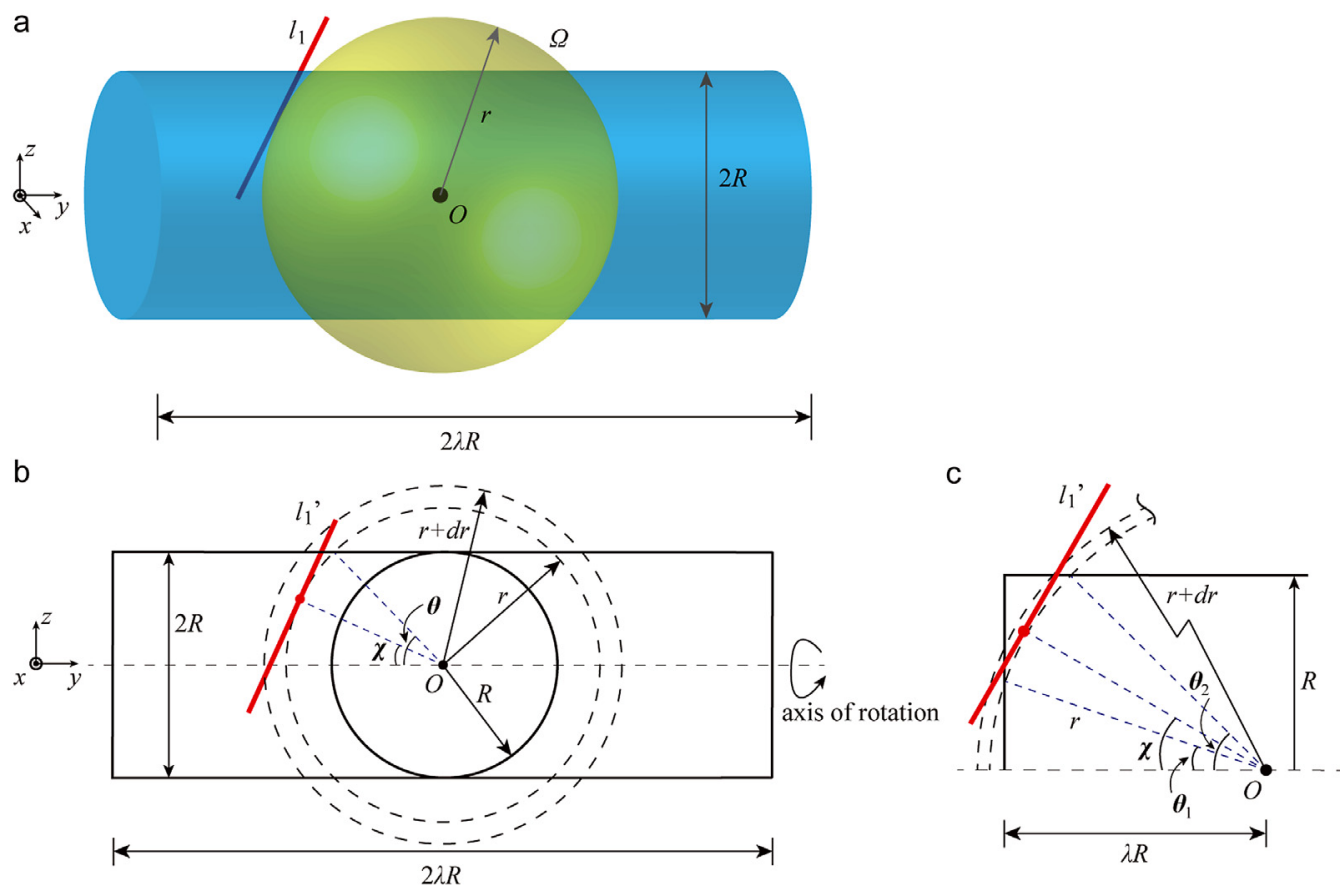

Fig. 5. Fibers in tangential contact with $\boldsymbol{\Omega}$ with contact points within a cylindrical NP. (a) Schematic showing a fiber $\left(l_{1}\right)$ in tangential contact with $\boldsymbol{\Omega}$ with contact point within the cylinder. (b) and (c) The projection of the cylinder and the fiber $\left(l_{1}{ }^{\prime}\right)$ on the $y z$-plane. $r$, the radius of $\boldsymbol{\Omega}$, is in the range of (b) $[R$, $\lambda R]$ and $(\mathrm{c})\left[\lambda R, \sqrt{\lambda^{2}+1} R\right]$.

contact and the contact points are outside the NP $\left(l_{4}\right)$ (Fig. 4). The average numbers of these fibers are denoted as $\bar{N}_{1}, \bar{N}_{2}$, $\bar{N}_{3}$ and $\bar{N}_{4}$, respectively. The expected total number $N$ of fibers in contact with the rod-like NP is

$$
\bar{N}=\bar{N}_{1}+\bar{N}_{2}+\bar{N}_{3}+\bar{N}_{4}
$$

which can be derived as follows.

First we deduce $\bar{N}_{1}$, the average number of fibers having tangential contact with $\Omega$ with contact points within the NP $\left(l_{1}\right)$ (Fig. 4(b)). Following Ogston (1958), for fibers in tangential contact with the spherical surface $\boldsymbol{\Omega}$, the number whose contact points fall within the region $[0, R]$ is $\gamma_{t} \cdot R^{2}$ where $\gamma_{t}=2 \pi v L, v$ being the average number of fibers per unit volume; the number of fibers whose contact points fall within the region $[r, r+d r]$ is $\gamma_{t} \cdot d\left(r^{2}\right)$. As shown in Fig. 5, the number of fibers whose contact points fall within $[r, r+d r]$ and the cylinder is (these fibers are all in contact with the cylinder.)

$$
\left\{\begin{array}{l}
\gamma_{t} \cdot d\left(r^{2}\right), r \in[0, R] \\
\gamma_{t} \cdot d\left(r^{2}\right) \cdot(1-\cos \theta), r \in[R, \lambda R] \\
\gamma_{t} \cdot d\left(r^{2}\right) \cdot\left(\cos \theta_{1}-\cos \theta_{2}\right), r \in\left[\lambda R, \sqrt{\lambda^{2}+1} R\right]
\end{array},\right.
$$

where $\cos \theta=\frac{\sqrt{r^{2}-R^{2}}}{r}, \cos \theta_{1}=\frac{\lambda R}{r}, \cos \theta_{2}=\frac{\sqrt{r^{2}-R^{2}}}{r}$.

Therefore, $\bar{N}_{1}$ is given by

$$
\begin{aligned}
\bar{N}_{1} & =\gamma_{t}\left[R^{2}+\int_{R}^{\lambda R}(1-\cos \theta) \cdot d r^{2}+\int_{\lambda R}^{\sqrt{\lambda^{2}+1} R}\left(\cos \theta_{1}-\cos \theta_{2}\right) \cdot d r^{2}\right] \\
& =\gamma_{t} \cdot f(\lambda) \cdot R^{2},
\end{aligned}
$$

where $f(\lambda)=\left(\sqrt{\lambda^{2}+1}-\lambda\right) \cdot \lambda+\ln \left(\sqrt{\lambda^{2}+1}+\lambda\right)$.

The deduction of $\bar{N}_{2}, \bar{N}_{3}$ and $\bar{N}_{4}$ is similar to that of $\bar{N}_{1}$ and can be found in Appendix A.

\subsection{Validation of theoretical model by Monte Carlo simulations}

To validate our theoretical model, Monte Carlo simulations were performed to evaluate the number of fibers in contact with spherical and cylindrical NPs in a network consisting of straight fibers.

A random network is generated in a cubic box with side length $a$. The fiber centers are randomly distributed in the box, and the fibers of length $2 L$ are oriented randomly. The total number of fibers in the box is $N_{f}$. Each fiber is discretized into 


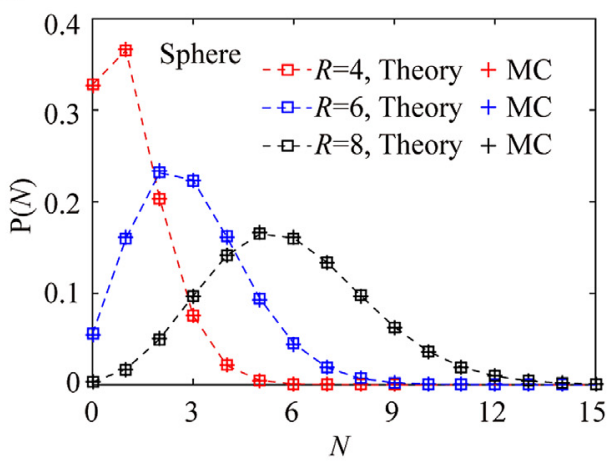

C

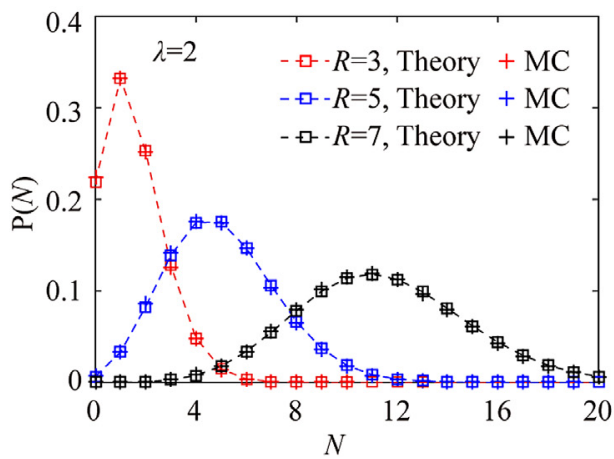

b

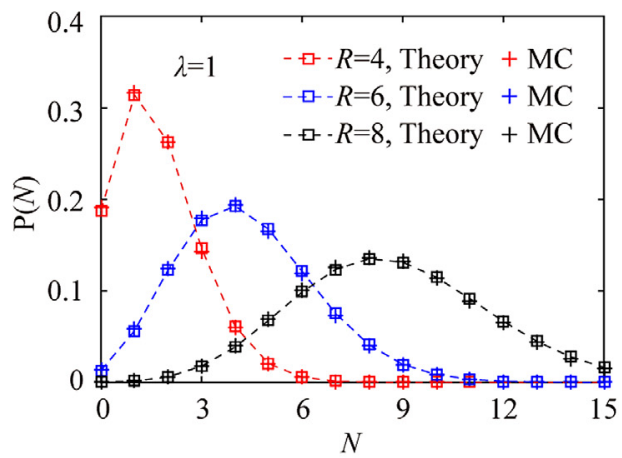

d

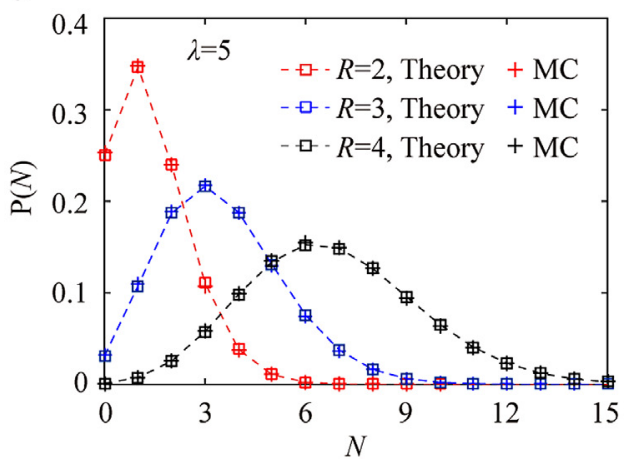

Fig. 6. Probability distribution of $N$, the number of fibers in contact with NPs, from the theoretical model and Monte Carlo simulations. (a) Results for spheres. (b)-(d) Results for cylinders with different ARs. The squares in color represent theoretical predictions by Eqs. (11) and (5) for NP with different radii, while the colored pluses represent Monte Carlo sampling results. The dashed lines are used to guide the line of sight. The parameters of the model system: $a=150 \sigma, v=1.28 \times 10^{-3} \sigma^{-3}, L=6 \sigma$ ( $\sigma$ is the unit of length).

a series of beads with a separation of $0.5 \sigma$, where $\sigma$ is the unit of length. The box is periodic, and the beads outside the box are mapped back into the box to ensure a constant fiber concentration in the system. In our simulations, the box size is $a=150 \sigma$, the product of fiber concentration $v$ and $L$ is $7.68 \times 10^{-3} \sigma^{-2}$, where the fiber concentration $v$ is expressed as $v=\frac{N_{f}}{a^{3}}$, and $L$ is changed from $3 \sigma$ to $18 \sigma$. To ensure the accuracy of the sampling, we generated ten random networks with different configurations.

A cylinder with radius $R$ and aspect ratio $\lambda$ was randomly placed in the random network. Then we counted the number of fibers in contact with the cylinder denoted by $N$. We repeated this procedure 1,000,000 times with ten different random networks to evaluate the distribution of $N$ and the probability when $N$ is equal to 0 .

The probability of no fibers in contact with a sphere in a fibrous network was derived by Ogston (1958). The model includes an assumption that the number of fibers in contact with a sphere, $N$, follows Poisson distribution (Chun et al., 1969; Rodbard and Chrambac, 1970). Thus the average number of fibers in contact with a sphere is given by

$$
\bar{N}=2 \pi v L R^{2}+\frac{4 \pi}{3} v R^{3}
$$

where $R$ is the radius of the sphere (Chun et al., 1969; Ogston, 1958). If $N$ follows Poisson distribution, then

$$
P(N=k)=\frac{\bar{N}^{k} \cdot \exp (-\bar{N})}{k !},
$$

and

$$
P(N=0)=\exp (-\bar{N})=\exp \left(-2 \pi v L R^{2}-\frac{4 \pi}{3} v R^{3}\right)
$$

Using Monte Carlo sampling, we obtain the probability distribution of $N$ for spheres with different radii in a model fiber network (Fig. 6(a)). It is seen that the simulation results agree well with the theoretical predictions from Eq. (11). For cylinders, Monte Carlo results are also consistent with the theoretical predictions by Eq. (5) (Fig. 6). Therefore, through our designed simulations, we prove that the number of fibers in contact with a sphere or cylinder follows Poisson distribution, and prove the accuracy of the theoretical model. In the following we would use Eq. (6) to obtain the probability of no fibers in contact with the cylinder. 
a

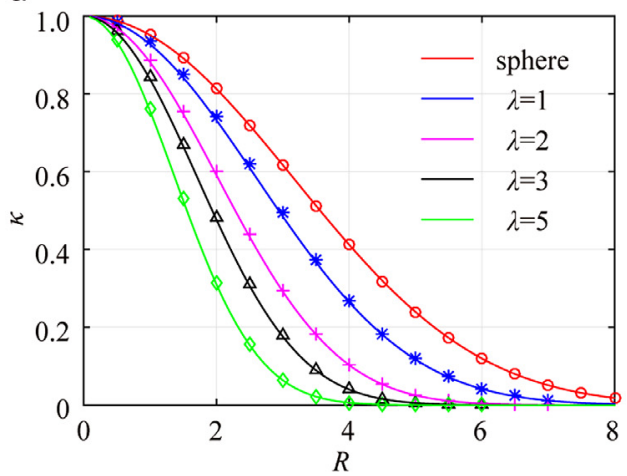

b

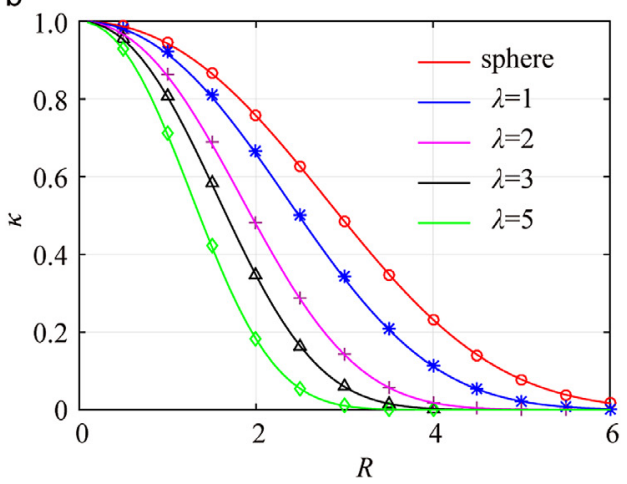

Fig. 7. The probability of no fibers in contact with NPs. The parameters of the model system: $a=150 \sigma, v L=7.68 \times 10^{-3} \sigma^{-3}$, (a) $L=18 \sigma$, (b) $L=3 \sigma$. Lines with different colors represent theoretical results of the spheres and the cylinders of different ARs, while symbols represent Monte Carlo results.
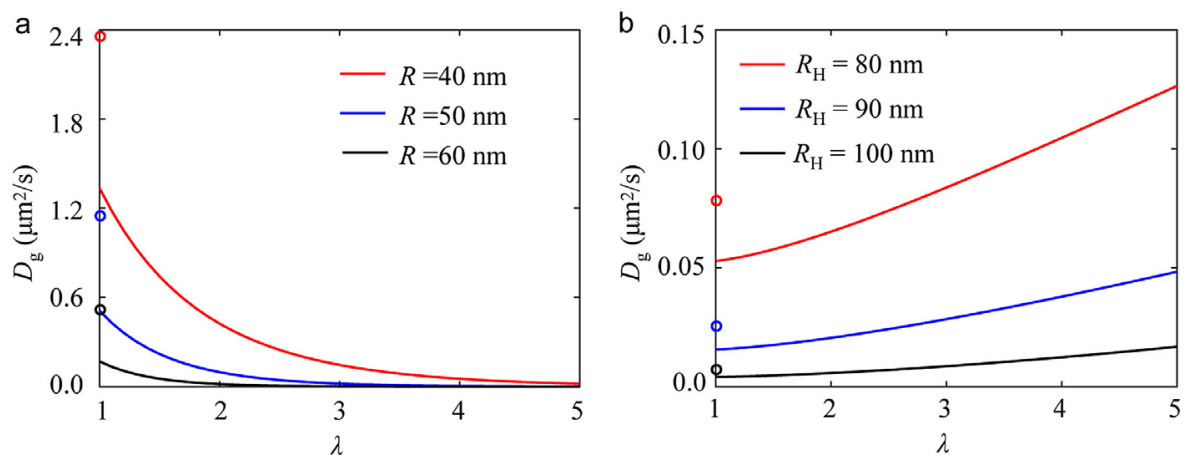

Fig. 8. Extended obstruction-scaling model for rod-like NPs in a non-adhesive gel. (a) Diffusivity $\left(D_{g}\right)$ of rod-like NPs with the same minor-axis diameter as a function of the aspect ratio $\lambda$. The circles represent the diffusivities of spherical NPs with the same diameter. $R$ is the radius of the NP. (b) Diffusivity ( $\left.D_{g}\right)$ of rod-like NPs with the same hydrodynamic diameter as a function of $\lambda$. The circles represent the diffusivities of spherical NPs of the same hydrodynamic size. $R_{H}$ is the hydrodynamic radius of the NPs. The parameters used in (a) and (b) are listed in Table 2.

We further compared the probability $\kappa$, theoretically predicted by Eqs. (12) and (6), with Monte Carlo simulations (Fig. 7). The results also agree very well, indicating the Eq. (6) could be utilized to evaluate the diffusivity of nanorods in porous medium.

\subsection{Diffusivity of nanorods in non-adhesive porous media}

We now investigate how the AR influences the diffusivity of nanorods with the same diameter but different lengths (Fig. 8(a)). The diffusivity decreases sharply as the rod length increases. For example, the diffusivities of nanorods with an AR of 2 are 0.32 -fold $(R=40 \mathrm{~nm}), 0.19$-fold $(R=50 \mathrm{~nm})$, and 0.10 -fold $(R=60 \mathrm{~nm})$ those of NPs with an AR of 1 (Fig. $8(\mathrm{a}))$. This prediction is consistent with the experimental observation of NPs diffusing in non-adhesive HEC. This is not surprising as the NPs with high AR are expected to have a large hydrodynamic diameter. We also compare the diffusivities of nanorods with the same hydrodynamic diameter. The diffusivity of the NPs is found to increase with the AR (Fig. 8(b)). Specifically, the diffusivities of nanorods with an AR of 5 are 2.4-fold $\left(R_{H}=80 \mathrm{~nm}\right), 3.1$-fold $\left(R_{H}=90 \mathrm{~nm}\right)$, and 4.2 -fold $\left(R_{H}=100 \mathrm{~nm}\right)$ those of NPs with an AR of 1 (Fig. 8(b)). The effect of the AR, as expected, becomes more significant as the NPs become longer and thinner. Note our results can also apply to gel electrophoresis and gel filtration of rod-like molecules (Rodbard and Chrambac, 1970; Viovy, 2000).

\section{Adhesive diffusion model}

\subsection{One-dimensional (1D) adhesive diffusion model}

To model the adhesive characteristics of mucus, we assume that there are discrete adhesion zones within the polymer solution, reflecting the fact that there exist heterogeneous hydrophobic and hydrophilic regions within the mucin fibers (Cone, 2009). We also assume that the NPs undergo hopping diffusion, i.e., they will be adsorbed to one adhesion region first (region A), and then detach after a while before being re-adsorbed to another adhesion region 


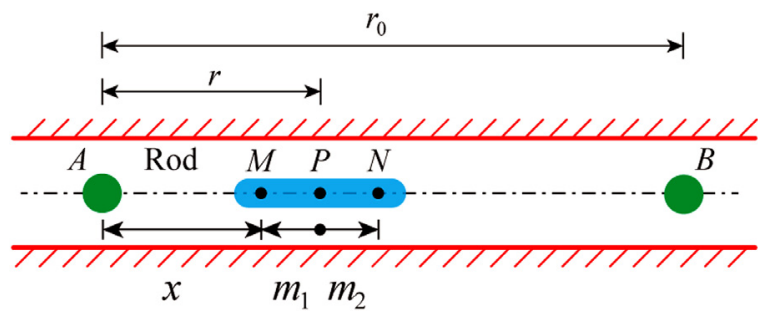

Fig. 9. Schematic showing NPs diffusion in a 1D cylindrical nanopore with adhesion regions A and B.

(Skaug et al., 2013; Walder et al., 2011) (region B), as shown in Fig. 3(b) and(c). The time for the NPs to move (hop) from region A to region $B$ can be calculated with the MFPT theory as follows.

We consider the diffusion of NPs in a 1D cylindrical nanopore with equally distributed adhesion regions at a spacing of $r_{0}$ (Figs. 3(b) and 9). For the purpose of mathematical manipulation, the interaction energy between the rod-like NPs per unit length and the adhesion region is described by the Morse potential

$$
V(x)=U_{0}\left(e^{-2 x / \rho}-2 e^{-x / \rho}\right),
$$

where $U_{0}$ is the adhesion strength, $x$ is the distance between one point on the NP and the adhesion region, and $\rho$ is the potential range, which is set to $20 \mathrm{~nm}$ (Fig. 3(d)). The interaction energy $U_{A}(r)$ between the whole NP and the adhesion region A can be expressed by the integral of the Morse potential, which is a function of $r$, the distance between the center of the NP and the adhesion region A (Fig. 9). The interaction energy between rod-like NPs and the adhesion region B is denoted by $U_{B}(r)$. The energy landscape when an NP moves from $A$ to $B$ is denoted by $U(r)$ which is the sum of $U_{A}(r)$ and $U_{B}(r)$

$$
U(r)=U_{A}(r)+U_{B}(r) .
$$

$U_{A}(r)$ and $U_{B}(r)$ are given by (Fig. 9)

$$
U_{A}(r)=\int_{0}^{L / 2} V(|A M|) d m_{1}+\int_{0}^{L / 2} V(|A N|) d m_{2},
$$

and

$$
U_{B}(r)=U_{A}\left(r_{0}-r\right),
$$

where $|A M|=\left|r-m_{1}\right|,|A N|=\left|r+m_{2}\right|$.

From Kramers' theory (Hanggi et al., 1990), the mean first passage time (MFPT) for a NP to move from A to B is given by

$$
t=\frac{1}{D_{g}} \cdot \int_{0}^{r_{0}} \exp \left(-\frac{U(r)}{k_{B} T}\right) \cdot\left[\int_{r}^{r_{0}} \exp \left(\frac{U(y)}{k_{B} T}\right) d y\right] \cdot d r
$$

where $D_{g}$ is the diffusivity of the NPs outside the adhesion region, $k_{B}$ is the Boltzmann constant, and $T$ is temperature. The effective diffusivity of the NPs in the 1D cylindrical nanopore is given by

$$
D_{e}=\frac{r_{0}^{2}}{2 t} \text {. }
$$

Setting $r_{0}=200 \mathrm{~nm}$, which is the typical pore size of the rat intestinal mucus, we obtain the energy landscape for rodlike NPs $(2 R=80 \mathrm{~nm}$ ) of different lengths when NPs move from region A to B (Fig. 10) and their diffusivities (Fig. 11(a)). When $U_{0} \geq 0.15 k_{B} T / \mathrm{nm}$, the diffusivity first increases then decreases with the rod length. NPs with a length of approximately $200 \mathrm{~nm}$ have the maximum diffusivity (Fig. 11(a)). For $U_{0}=0.15 \mathrm{k}$ T/nm, the diffusivity of NPs with a length of $200 \mathrm{~nm}$ is 11.0-fold that of NPs with a length of $80 \mathrm{~nm}$. Adhesion (only a few $\mathrm{k}_{\mathrm{B}} \mathrm{T}$ (Smart, 2005)) can significantly affect the diffusivity of NPs in polymer gels, resulting in higher diffusivity for NPs of a certain length. When $U_{0}=0$, this model becomes the obstruction-scaling model, i.e., $D_{e}=D_{g}$, and the diffusivity decreases sharply with increasing NP length (Figs. 11(a) and $8(\mathrm{a})$ ).

\subsection{Three-dimensional (3D) adhesive diffusion model}

For the 3D case, we construct 3D orthonormal regular networks with cross-linking points as the adhesion regions (Fig. 3(c)). The distance between the adjacent adhesion regions A and B is $r_{0}$, the average time for NPs to move from A to $\mathrm{B}$ is $t$, and the effective diffusivity of the NPs is

$$
D_{e}=\frac{r_{0}^{2}}{6 t}
$$




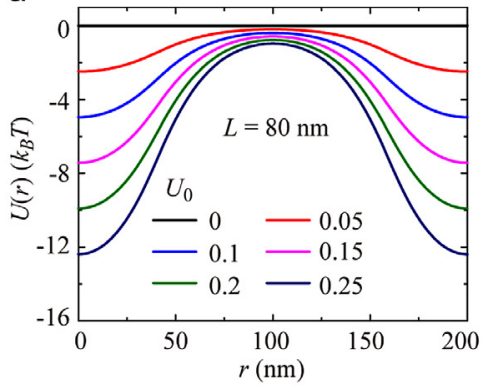

d

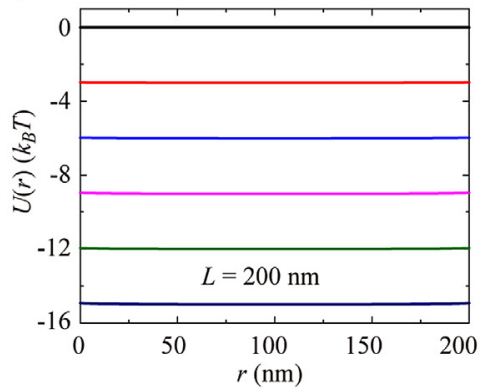

b

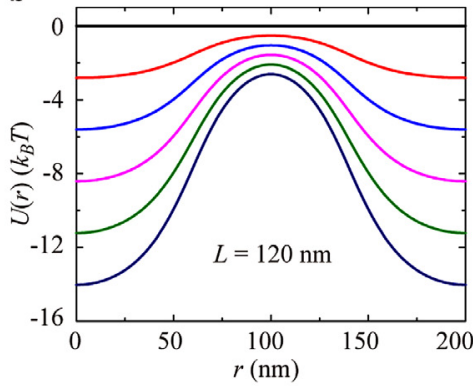

$\mathrm{e}$

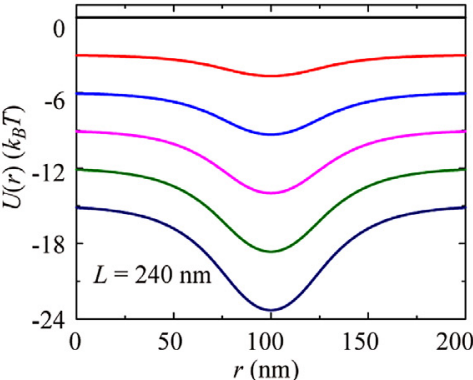

C

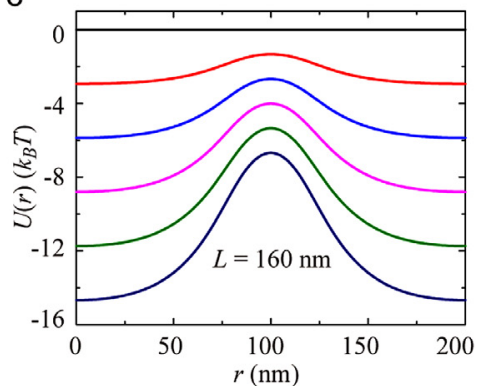

f

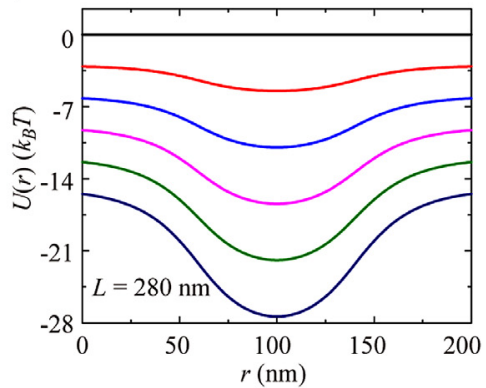

Fig. 10. Energy landscape for rod-like NPs of different lengths to move from region A to B (1D). (a)-(f) NPs with diameter of $2 R=80$ nm and lengths of $80 \mathrm{~nm}, 120 \mathrm{~nm}, 160 \mathrm{~nm}, 200 \mathrm{~nm}, 240 \mathrm{~nm}$ and $280 \mathrm{~nm}$, respectively. $U_{0}$ is the adhesion strength with a unit of $\mathrm{k}_{\mathrm{B}} \mathrm{T} / \mathrm{nm}$, the potential range $\rho$ is set to $20 \mathrm{~nm}$.
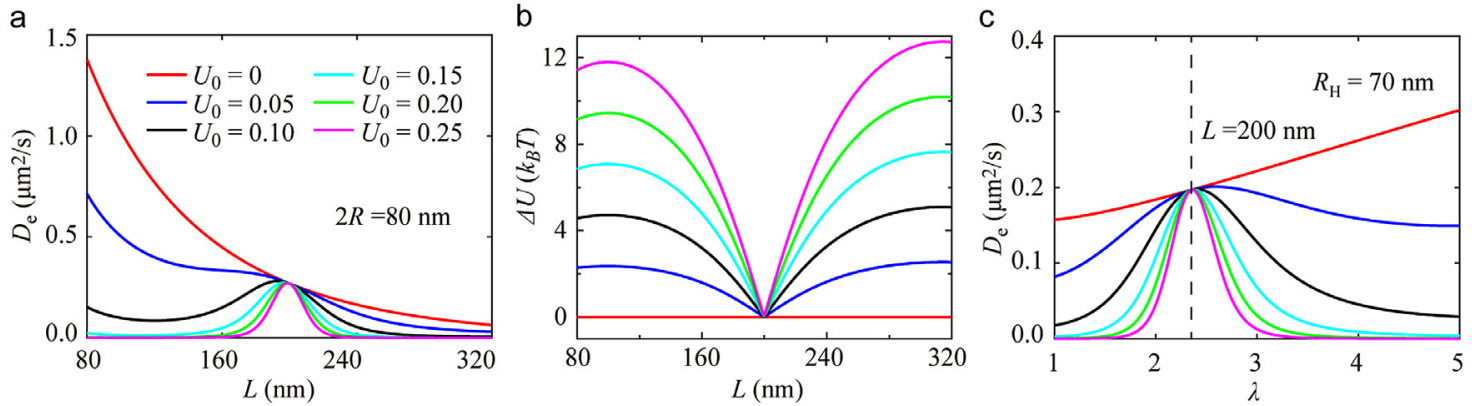

Fig. 11. Diffusivity of NPs in adhesive porous media (1D). (a) Effective diffusivity $\left(D_{e}\right)$ of NPs in $1 \mathrm{D}$ nanopores at different adhesion strengths as a function of the NP length, $L$. The diameter of the NPs considered is $80 \mathrm{~nm}$. (b) Energy barrier when rod-like NPs of different lengths move from A to B, which is defined as the difference between the maximum and the minimum potential energies. (c) Effective diffusivity of rod-like NPs with the same hydrodynamic radius $\left(R_{H}=70 \mathrm{~nm}\right)$ in $1 \mathrm{D}$ nanopores as a function of $\lambda$. In all cases, the distance between the adjacent adhesion regions $\mathrm{A}$ and $\mathrm{B}$ is $r_{0}=200 \mathrm{~nm}$.

For the convenience of mathematical derivation of the MFPT, we assume that there is a spherical absorbing wall $\Sigma$ with a radius of $r_{0}$ around the adhesion zone A, and $t$ is approximately given by the MFPT for a NP to move from A to $\Sigma$ (Fig. 12). The energy landscape when a NP move from A to $\Sigma$ is denoted by $U(r)$, where $r$ is the distance between the center of the NP and the adhesion region A. We will obtain the MFPT for a NP to move from A to $\Sigma$ as follows.

\subsubsection{MFPT without external potential}

In order to solve the problem, we suppose there is an invisible hand at the absorbing wall (Maxwell's demon). As long as the NP is captured at $r_{0}$, it will be instantly placed back to the origin and start to diffuse again. If we think of a large number of similar systems (ensemble), we can speak of a steady state flux from the origin toward the absorbing wall. The flux can be described as a probability distribution function $p(r)$ which gives a steady-state flux as

$$
j=-D \cdot \frac{\partial p}{\partial r} \cdot 4 \pi r^{2}=\text { constant }
$$

For the absorbing wall, the boundary condition is

$$
p\left(r_{0}\right)=0
$$




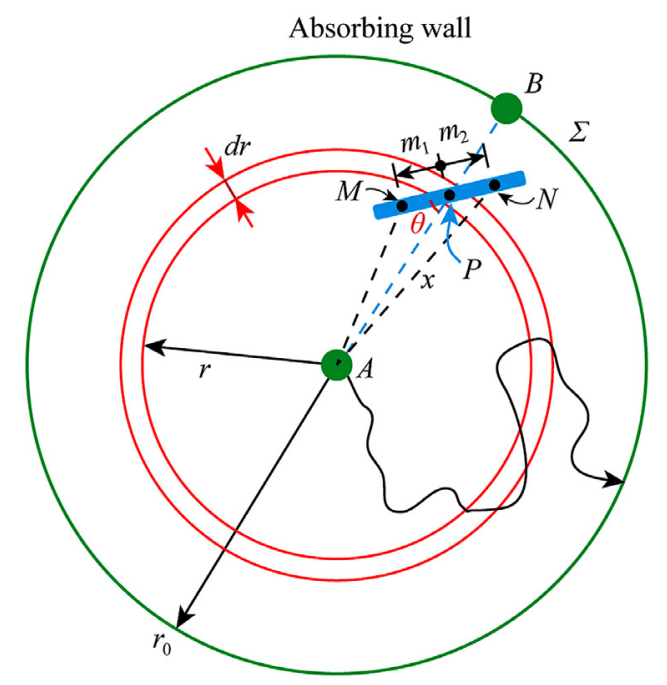

Fig. 12. Schematic showing the diffusion of NPs from the adhesion region A to the spherical absorbing wall $\Sigma$.

and a probability distribution function must satisfy

$$
\int_{0}^{r_{0}} p(r) \cdot 4 \pi r^{2} \cdot d r=1
$$

Integrating $j=-D \cdot \frac{\partial p}{\partial r} \cdot 4 \pi r^{2}$ from $r$ to $r_{0}$ and using $p\left(r_{0}\right)=0$, we have

$$
p(r)=\frac{j}{4 \pi D} \cdot\left(\frac{1}{r}-\frac{1}{r_{0}}\right) .
$$

Integrating again from 0 to $r_{0}$ and using $\int_{0}^{r_{0}} p(r) \cdot 4 \pi r^{2} \cdot d r=1$ yields the steady state flux (number of NPs captured per unit time) as

$$
j=\frac{6 D}{r_{0}^{2}} \text {. }
$$

The time for one NP to travel from 0 to $r_{0}$ is therefore

$$
t=\frac{1}{j}=\frac{r_{0}^{2}}{6 D}
$$

which is the mean first passage time.

\subsubsection{MFPT in the presence of an external force/potential}

Next we address the MFPT of a NP in the presence of a potential $U(r)$ which exerts a force on the particle,

$$
F(r)=-\frac{d U(r)}{d r} \text {. }
$$

Due to the presence of an external force, the particle flux is modified to

$$
j=-D \cdot \frac{\partial p}{\partial r} \cdot 4 \pi r^{2}+v \cdot p \cdot 4 \pi r^{2},
$$

where $v=\frac{F}{\xi}$ is the drift velocity of the particle due to the force $F, \xi$ being the drag coefficient. Therefore,

$$
j=-D \cdot \frac{\partial p}{\partial r} \cdot 4 \pi r^{2}-\frac{1}{\xi} \cdot p \cdot 4 \pi r^{2} \cdot \frac{d U(r)}{d r} .
$$

According to the Einstein relation

$$
D \xi=k_{B} T,
$$

we can rewrite the above equation as

$$
j=-D \cdot 4 \pi r^{2}\left(\frac{\partial p}{\partial r}+\frac{p}{k_{B} T} \cdot \frac{d U(r)}{d r}\right)=\text { constant. }
$$


Multiplying both sides by $\exp \left(\frac{U}{k_{B} T}\right)$,

$$
j \cdot \exp \left(\frac{U}{k_{B} T}\right)=-D \cdot 4 \pi r^{2}\left(\exp \left(\frac{U}{k_{B} T}\right) \cdot \frac{\partial p}{\partial r}+\frac{p}{k_{B} T} \cdot \exp \left(\frac{U}{k_{B} T}\right) \cdot \frac{d U(r)}{d r}\right)=-D \cdot 4 \pi r^{2} \frac{d}{d r}\left[p \cdot \exp \left(\frac{U}{k_{B} T}\right)\right],
$$

then integrating from $r$ to $r_{0}$ while noting $p\left(r_{0}\right)=0$ gives

or

$$
j \cdot \int_{r}^{r_{0}} \frac{1}{4 \pi y^{2}} \cdot \exp \left(\frac{U(y)}{k_{B} T}\right) d y=D \cdot p(r) \cdot \exp \left(\frac{U(r)}{k_{B} T}\right),
$$

$$
p(r)=\frac{j}{D} \cdot \exp \left(-\frac{U(r)}{k_{B} T}\right) \cdot \int_{r}^{r_{0}} \frac{1}{4 \pi y^{2}} \cdot \exp \left(\frac{U(y)}{k_{B} T}\right) d y .
$$

Integrating again from 0 to $r_{0}$ and using $\int_{0}^{r_{0}} p(r) \cdot 4 \pi r^{2} \cdot d r=1$, we obtain

$$
D=j \cdot \int_{0}^{r_{0}} \exp \left(-\frac{U(r)}{k_{B} T}\right) \cdot r^{2} \cdot\left[\int_{r}^{r_{0}} \frac{1}{y^{2}} \cdot \exp \left(\frac{U(y)}{k_{B} T}\right) d y\right] \cdot d r .
$$

Similar to the free diffusion case, the MFPT is identified to be

$$
t=\frac{1}{j}=\frac{1}{D} \cdot \int_{0}^{r_{0}} \exp \left(-\frac{U(r)}{k_{B} T}\right) \cdot r^{2} \cdot\left[\int_{r}^{r_{0}} \frac{1}{y^{2}} \cdot \exp \left(\frac{U(y)}{k_{B} T}\right) d y\right] \cdot d r .
$$

When $U(r)=0$ (i.e., in the absence of any external potential) or $U(r)=$ constant (i.e., in the absence of any external force, $f(r)=0$ ), the above formula gives

$$
t=\frac{1}{j}=\frac{1}{D} \cdot \int_{0}^{r_{0}} r^{2} \cdot\left(\frac{1}{r}-\frac{1}{r_{0}}\right) \cdot d r=\frac{r_{0}^{2}}{6 D}
$$

which is consistent with the result of the free diffusion case.

\subsubsection{Energy landscape $\mathrm{U}(\mathrm{r})$ for a NP to move from $A$ to $\Sigma$}

The interaction energy between rod-like NPs per unit length and the adhesion region is also described by Morse potential shown in Eq. (13). The interaction energy $U_{A}(r, \theta)$ between the whole NP and the adhesion region $A$ can be expressed by the integral of the Morse potential, which is a function of $r$, the distance between the center of the NP and the adhesion region A, and $\theta$, the orientation of the NP (Fig. 12). The interaction energy between rod-like NPs and the spherical absorbing wall, $\Sigma$, can be represented by the interaction energy, $U_{B}(r, \theta)$, between rod-like NPs and the adhesion region B. The potential energy, $U(r, \theta)$, is the sum of the interaction energy between the NP and the adhesion regions A and B

$$
U(r, \theta)=U_{A}(r, \theta)+U_{B}(r, \theta) .
$$

$U_{A}(r, \theta)$ and $U_{B}(r, \theta)$ are given by (Fig. 12)

$$
U_{A}(r, \theta)=\int_{0}^{L / 2} V(|A M|) d m_{1}+\int_{0}^{L / 2} V(|A N|) d m_{2},
$$

where $|A M|=\sqrt{r^{2}+m_{1}^{2}-2 \cdot r \cdot m_{1} \cdot \cos \theta},|A N|=\sqrt{r^{2}+m_{2}{ }^{2}+2 \cdot r \cdot m_{2} \cdot \cos \theta}$, and

$$
U_{B}(r, \theta)=U_{A}\left(r_{0}-r, \theta\right) \text {. }
$$

For a given $r$, the probability density of $\theta$ is

$$
P(r, \theta)=\frac{1}{Z(r, \theta)} \cdot \exp (-U(r, \theta)) \cdot \sin \theta,
$$

where $Z(r, \theta)$ is the partition function and can be expressed as

$$
Z(r, \theta)=\int_{0}^{\pi / 2} \exp (-U(r, \theta)) \cdot \sin \theta \cdot d \theta .
$$

For a given $r$, the mean potential energy $U(r)$ is

$$
U(r)=\int_{0}^{\pi / 2} U(r, \theta) \cdot P(r, \theta) \cdot d \theta .
$$

Combining Eqs. (36)-(40), we obtain the energy landscape $U(r)$ for a NP to move from A to $\Sigma$. By substituting Eq. (40) into Eq. (34) and replacing $D$ with $D_{g}$, which is the diffusivity of the NPs outside the adhesion region and taken as the diffusivity of the NPs in non-adhesive gels, we can get the MFPT for a NP to diffuse from $A$ to $\Sigma$ as

$$
t=\frac{1}{D_{g}} \cdot \int_{0}^{r_{0}} \exp \left(-\frac{U(r)}{k_{B} T}\right) \cdot r^{2} \cdot\left[\int_{r}^{r_{0}} \frac{1}{y^{2}} \cdot \exp \left(\frac{U(y)}{k_{B} T}\right) d y\right] \cdot d r,
$$


a

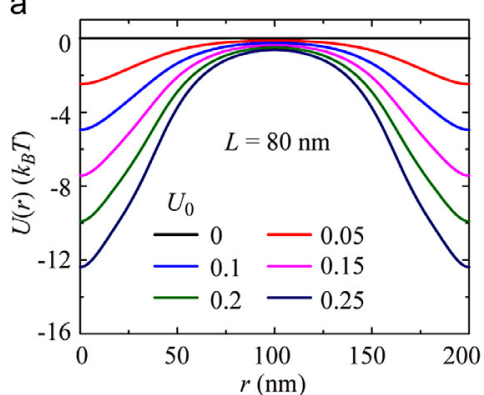

d

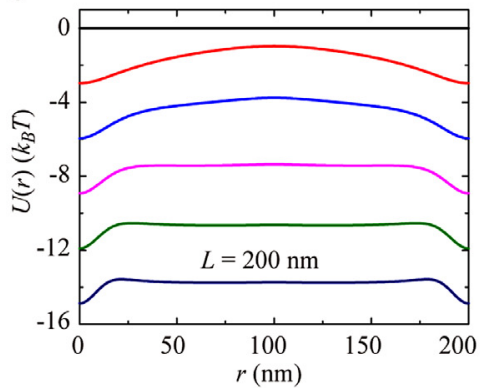

$\mathrm{b}$

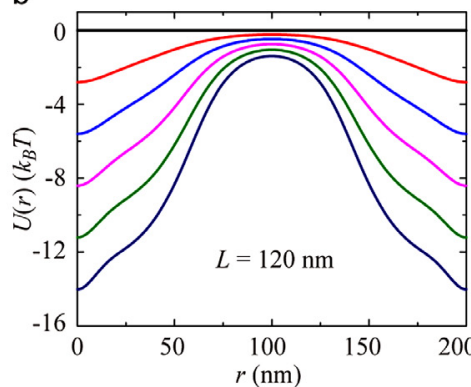

e

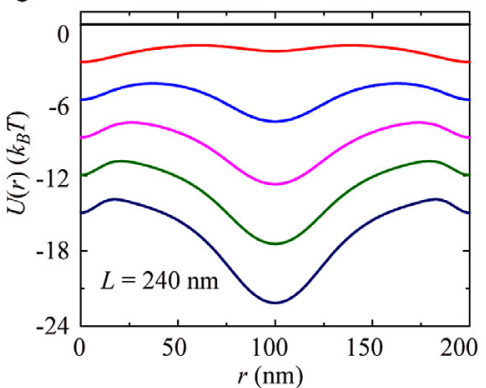

C

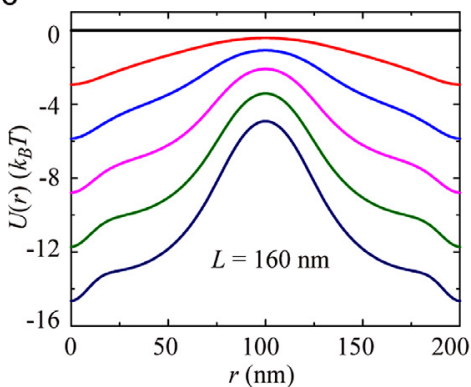

$f$

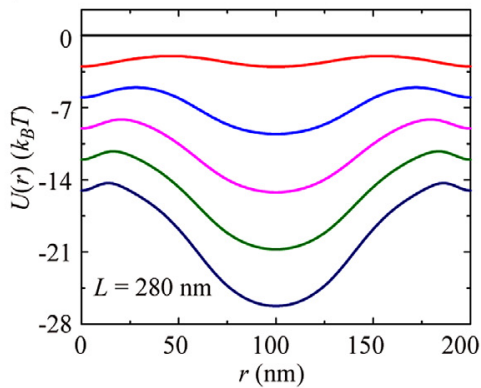

Fig. 13. Energy landscape for rod-like NPs of different lengths when NPs move from $A$ to $\Sigma$ (3D). (a)-(f) NPs with a length of $80 \mathrm{~nm}, 120 \mathrm{~nm}$, $160 \mathrm{~nm}$, $200 \mathrm{~nm}, 240 \mathrm{~nm}$, and $280 \mathrm{~nm}$, respectively. $U_{0}$ is the adhesion strength with a unit of $\mathrm{k}_{\mathrm{B}} \mathrm{T} / \mathrm{nm}$, the potential range $\rho$ is set to 20 nm. The diameter of the NPs is $80 \mathrm{~nm}$.
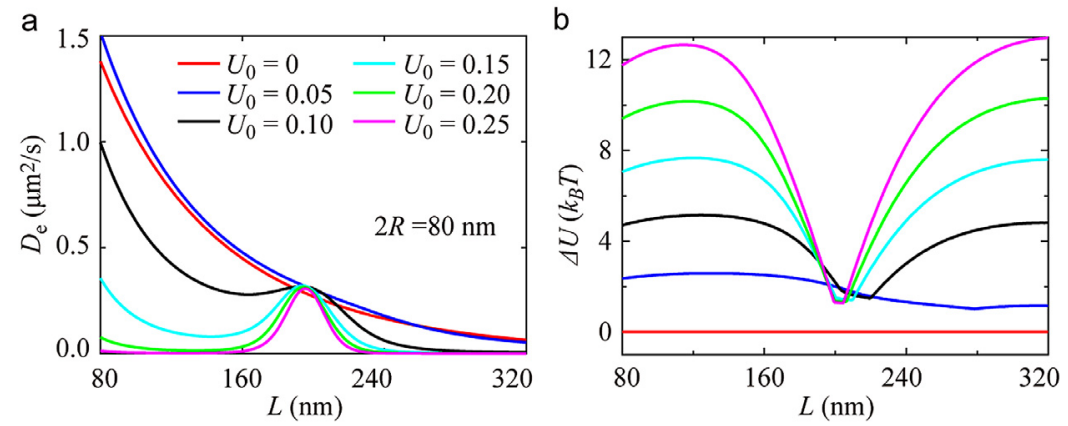

Fig. 14. Diffusivity of NPs in adhesive porous media (3D). (a) Effective diffusivity $\left(D_{e}\right)$ of NPs in 3D porous media at different adhesion strengths, plotted against the NP length, $L$. The diameter of the NPs considered is $80 \mathrm{~nm}$. (b) Energy barrier when rod-like NPs of different lengths move from A to B, which is defined as the difference between the maximum and the minimum potential energies. In all cases, the distance between the adjacent adhesion regions $\mathrm{A}$ and $\mathrm{B}$ is $r_{0}=200 \mathrm{~nm}$.

\subsubsection{Diffusivity of rod-like NPs in adhesive porous media}

Given the adhesion strength of $U_{0}$, we obtain the energy landscape (Fig. 13), MFPT values for NPs with different lengths and, accordingly, their effective diffusivity (Fig. 14(a)). For rod-like NPs with a diameter of $2 R=80 \mathrm{~nm}$, when $U_{0} \geq 0.15 \mathrm{k}_{\mathrm{B}} \mathrm{T} / \mathrm{nm}$, the diffusivity of NPs reaches a maximum at NP length of $200 \mathrm{~nm}$. When $U_{0}=0$ and $U_{0}=0.05 \mathrm{k}_{\mathrm{B}} \mathrm{T} / \mathrm{nm}$, the diffusivity decreases with increasing NP length (Fig. 14(a)). These results are similar to those in the 1D case and clearly show that NPs of a certain length diffuse fastest in mucus.

The observed variation in the position of the diffusivity peak with the length of the NPs could be explained by the depth of the energy barrier during NP diffusion, as shown in Fig. 14(b) for the 3D case and in Fig. 11(b) for the 1D case. In the presence of adhesion (e.g., $U_{0}=0.2 \mathrm{k}_{\mathrm{B}} \mathrm{T} / \mathrm{nm}$ ), the energy profile has a deep well located in the adhesion region for short nanorods, indicating that these nanorods will be trapped by the attraction of the adhesion region where they reside (Figs. 10(a)-(c) and 13(a)-(c)). In contrast, for overly long nanorods, the deep well lies outside the adhesion regions (Figs. 10(f) and 13(f)), indicating that these NPs will be trapped by the attraction of two adhesion regions. Only for nanorods with intermediate length does the energy landscape exhibit a shallow well, suggesting a low energy barrier to NP transport (Figs. 13(d) and 14(b)). At such a length, the NPs can experience attraction from adjacent adhesion region, thereby reducing the energy barrier. 

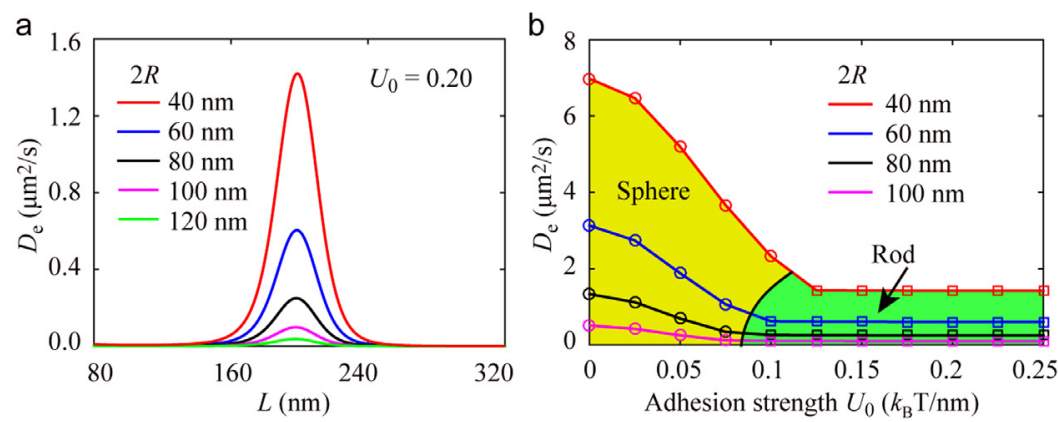

Fig. 15. Diffusivity of NPs of different diameters. (a) Effective diffusivity $\left(D_{e}\right)$ of NPs of different diameters in 1D nanopores when the adhesion strength $U_{0}=0.2 \mathrm{k}_{\mathrm{B}} \mathrm{T} / \mathrm{nm}$. (b) Maximum effective diffusivity for nanorods with the same minor-axis diameter at different adhesion strengths. The squares represent NPs with length similar to the spacing between the adhesion regions $\left(r_{0}\right)$, and the circles represent NPs with $\lambda=1$.

We also calculate the diffusivity of NPs with the same hydrodynamic diameter, as shown in Fig. 11(c). Given $R_{H}=70 \mathrm{~nm}$, the rod becomes long and thin as the AR, $\lambda$, increases. It seems that in the presence of adhesion, the profile of diffusivity as a function of length is not monotonic. There also exists an optimal length at which NPs exhibit maximum diffusivity (Fig. 11(c)).

In addition, we obtain the diffusivity of nanorods with different diameters given the adhesion strength $U_{0}=0.2 \mathrm{k}_{\mathrm{B}} \mathrm{T} / \mathrm{nm}$. The results show that nanorods with a length of $\sim 200 \mathrm{~nm}$ (close to the separation distance between adhesion regions, $r_{0}$ ) achieve the maximum diffusivity regardless of the NP diameter (Fig. 15(a)). The optimal diffusivities of nanorods with the same minor-axis diameter at different adhesion strengths are shown in Fig. 15(b). With increasing adhesion strength, the profiles of the diffusivities decrease first, then tend to become flat, and the NPs with the optimal diffusivity shift from nanospheres to nanorods (with length near $r_{0}$ ). Thus, for NPs diffusing in a porous medium with discrete adhesion regions, nanorods whose length is comparable to the spacing of the adhesion regions can achieve high diffusivity.

\subsection{Discussions}

In the theoretical model, we have assumed that the NPs hop from one adhesive region to the next during diffusion in adhesive mucus. To verify the hopping experimentally, we performed additional tracking studies under stimulated emission depletion (STED) microscopy. White gridlines were added to the STED images to aid the tracking of the displacements of the particles. As indicated by the arrowheads in Fig. S1 and Video 1 in Supplementary material, two nanorods exhibited sudden shifts in position at certain time points, which suggests hopping diffusion. In comparison, no such hopping phenomenon was observed during nanorod diffusion in HEC (Fig. S2 and Video 2 in Supplementary material).

In this work, we have assumed that the cross-linked sites in adhesive gels are adhesion regions that interact with NPs, while the others are non-interactive. The interactive and non-interactive sites could be switched in our theoretical model without fundamentally influencing our results. This means that the heterogeneous interactions of polymers with NPs play key roles in the superior diffusivity of nanorods. If the NPs experience homogeneous interactions throughout the whole media, that is, if $U(r)$ is a constant along the fiber (i.e., the external force $f(r)=0$ ), then the adhesive case reduces to the non-adhesive case $\left(D_{e}=D_{g}\right.$, Eq. (35)). This reduction suggests that in a medium lacking heterogeneous interactions, the nanorods should not diffuse faster than spherical NPs with the same minor-axis diameter.

The interaction energy between the whole NP and the adhesion region, $V(r)$, can also be described by a piecewise linear potential function with a well depth of $U$ (Fig. 16(b)), which is expressed as

$$
V(r)=\left\{\begin{array}{l}
-U, r \leq L / 2 \\
-U \cdot\left(1-\frac{r-L / 2}{m}\right), L / 2<r \leq L / 2+m, \\
0, r>L / 2+m
\end{array}\right.
$$

where $L$ is the length of NPs, $m$ is potential range. We assume that the potential range is less than half the separation of the adhesion regions, i.e. $m<r_{0} / 2$. The energy landscapes, $V(r)$, when NPs of different lengths move from adhesion region A to B are shown in Fig. 16, it can be represented by the piecewise linear potential function shown in Fig. 16(g), which is expressed as

$$
V(r)=U \cdot\left\{\begin{array}{l}
-1,0 \leq r \leq a \\
\frac{1-k}{b} \cdot r-\left[1+(1-k) \cdot \frac{a}{b}\right], a \leq r \leq a+b \\
-k, a+b \leq r \leq a+b+c \\
-\frac{1-k}{b} \cdot r+\frac{1}{b} \cdot[(a+b+c)-k(a+2 b+c)], a+b+c \leq r \leq a+2 b+c \\
-1, a+2 b+c \leq r \leq 2 a+2 b+c
\end{array},\right.
$$




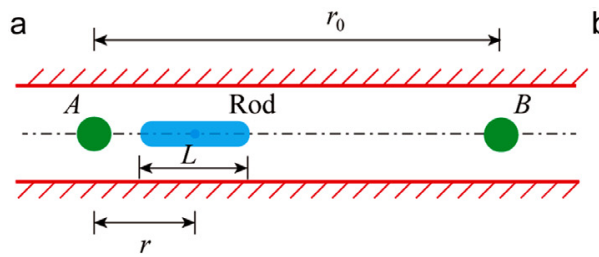

b

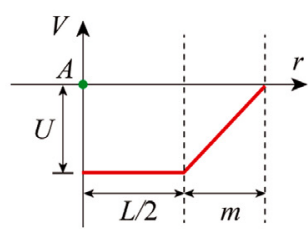

C

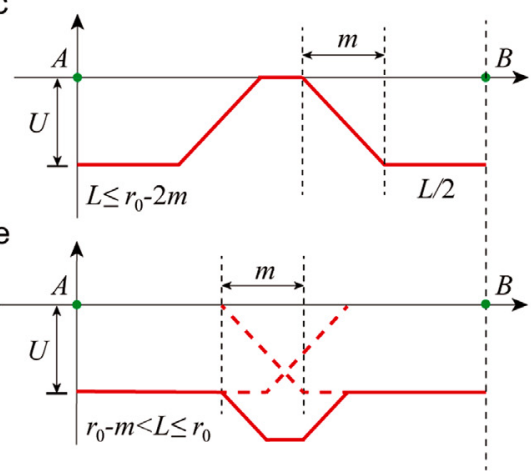

g

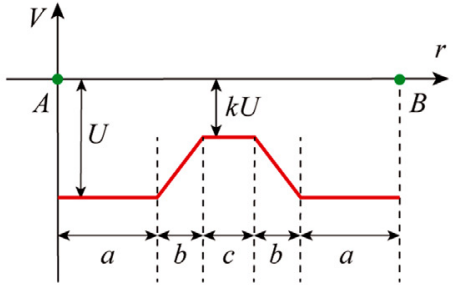

d

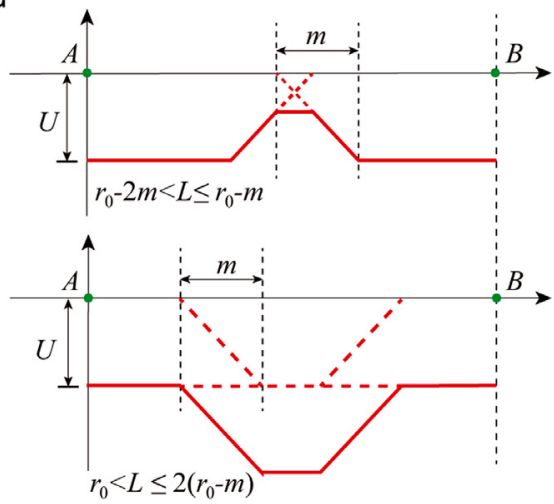

h

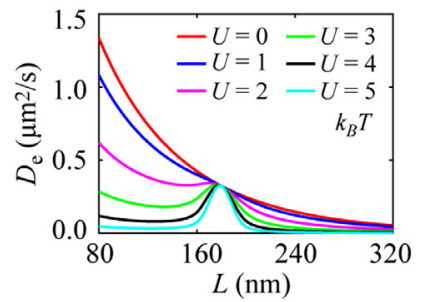

Fig. 16. Energy landscape when NPs of different lengths move from adhesion region A to B, and the diffusivity of NPs. (a) Schematic showing NPs diffusion in $1 \mathrm{D}$ cylindrical nanopores with adhesion regions A and B. (b) Interaction potential between the NPs and the adhesion region as a function of distance. $L$ is the length of NPs, $m$ the potential range, $U$ the potential well depth. (c)-(f) Energy landscapes when NPs of different lengths move from adhesion region A to B. (g) Schematic showing the energy landscape when NPs diffuse from adhesion region A to B. (h) Effective diffusivity of NPs in 1D nanopores at different adhesion strength, plotted against the NPs length, $L$. The diameter of the NPs considered is $80 \mathrm{~nm}$, the potential range $m$ is set to $20 \mathrm{~nm}$.

where $a, b, c$ and $k$ are parameters shown in Fig. 16(g). The values of $a, b, c$ and $k$ can be given by

$$
\left\{\begin{array}{l}
a=L / 2 \\
b=m \\
c=r_{0}-L-2 m \\
k=0
\end{array},\right.
$$

when $L \leq r_{0}-2 m$;

$$
\left\{\begin{array}{l}
a=L / 2 \\
b=r_{0}-L-m \\
c=L+2 m-r_{0} \\
k=\left(2 m+L-r_{0}\right) / m
\end{array},\right.
$$

when $r_{0}-2 m<L \leq r_{0}-m$;

$$
\left\{\begin{array}{l}
a=r_{0}-m-L / 2 \\
b=m+L-r_{0} \\
c=r_{0}-L \\
k=\left(2 m+L-r_{0}\right) / m
\end{array},\right.
$$


when $r_{0}-m<L \leq r_{0}$

$$
\left\{\begin{array}{l}
a=r_{0}-m-\frac{L}{2} \\
b=m \\
c=L-r_{0} \\
k=2
\end{array},\right.
$$

when $r_{0}<L \leq 2\left(r_{0}-m\right)$.

Given the energy landscapes $V(r)$ for a NP moving from one adhesion region A to the adjacent adhesion $\mathrm{B}$, the mean first passage time (MFPT) for a NP to diffuse from A to B is given by:

$$
t=\frac{1}{D} \int_{0}^{2 a+2 b+c} \exp \left[-\frac{V(x)}{k_{B} T}\right] \cdot\left\{\int_{x}^{2 a+2 b+c} \exp \left[\frac{V(y)}{k_{B} T}\right] \cdot d y\right\} \cdot d x
$$

where $D$ is diffusivity of NPs. Substituting Eq. (43) into Eq. (45), $t$ can be expressed as

$$
\begin{aligned}
t= & \frac{1}{D}\left\{\left[\frac{2 a b+b c}{(1-k) \bar{U}}+\frac{2 b^{2}}{(1-k)^{2} \bar{U}^{2}}+a c\right] \exp [(1-k) \bar{U}]-\left[\frac{2 a b+b c}{(1-k) \bar{U}}-\frac{2 b^{2}}{(1-k)^{2} \bar{U}^{2}}-a c\right] \exp [-(1-k) \bar{U}]\right\} \\
& +\frac{1}{D}\left[2 a^{2}+\frac{c^{2}}{2}-\frac{4 b^{2}}{(1-k)^{2} \bar{U}^{2}}\right],
\end{aligned}
$$

with $\bar{U}=\frac{U}{k_{B} T}$ when $k \neq 1$, and

$$
t=\frac{1}{2 D} \cdot(2 a+2 b+c)^{2}
$$

when $k=1$.

Consider rod-like NPs with a diameter of $2 R=80 \mathrm{~nm}$, we obtained the effective diffusivity of these NPs with different lengths, as shown in Fig. 16(h), which are similar to those when the interaction is described by the Morse potential, indicating that the model does not depend on one specific potential, indicative of the generality of our results.

\section{Conclusions}

We have conducted a joint experimental and theoretical study of the physical mechanisms that underlie anomalous diffusion of rod-like nanoparticles in mucus, an adhesive biological polymeric gel of key importance to drug delivery systems. By extending the so-called obstruction-scaling model to the diffusion of non-spherical solute particles, we demonstrate that the diffusivity of NPs in non-adhesive porous media would increase monotonically with the aspect ratio of nanorods with the same hydrodynamic diameter and decrease with the aspect ratio of nanorods with the same minor-axis diameter. Experimental results based on the diffusion of NPs in HEC solution verify our theoretical predictions. In contrast, in an adhesive porous medium, we find that the aspect ratio of the NPs and their adhesive interactions with the host polymer play key roles in tuning the diffusivity of the nanorods, resulting in an optimal aspect ratio at which the NPs achieve the maximum diffusivity. By incorporating the matrix-NPs interactions into the obstruction-scaling model via the MFPT theory, it is shown that such anomalous diffusion can be attributed to adhesion-induced hopping of NPs in the adhesive porous gel. Our theoretical models and predictions have been validated through STED microscopy and measurements of NPs diffusion in mucus as a typical model system of an adhesive polymer solution and in HEC as a typical model system of a non-adhesive polymer solution. Our study provides physical insights into the anomalous diffusion behaviors of rod-like NPs in biological systems. The developed theoretical models provide guidelines for a range of applications involving particle diffusion in complex porous media, with a prominent example being the development of efficient drug delivery system.

\section{Acknowledgments}

We are grateful for financial support from the National Natural Science Foundation of China (11422215, 11272327 and 11672079 to X.S., 81373356, 81573378 and 8177130638 to Y.G.), the "Science and technology innovation action plan for basic research" of Shanghai 2014 (14JC1493200 to Y.G.), and the National Science Foundation under Grant CMMI-1562904 (H.G.). This work was also partly supported by CASIMM0120153020 and Opening fund of State Key Laboratory of Nonlinear Mechanics. The computation is mainly supported by the Supercomputing Center of Chinese Academy of Sciences (SCCAS).

\section{Appendix A. Average number of fibers in contact with rod-like NPs}

\section{A.1. Fibers in end contact with $\Omega$ with contact points within the NP}

Here we deduce $\bar{N}_{2}$, the average number of fibers having end contact with $\Omega$ with contact points within the NP $\left(l_{2}\right)$ (Fig. 4(b)). For fibers that are defined as end contact with the spherical surface $\boldsymbol{\Omega}$, the number whose contact points fall 

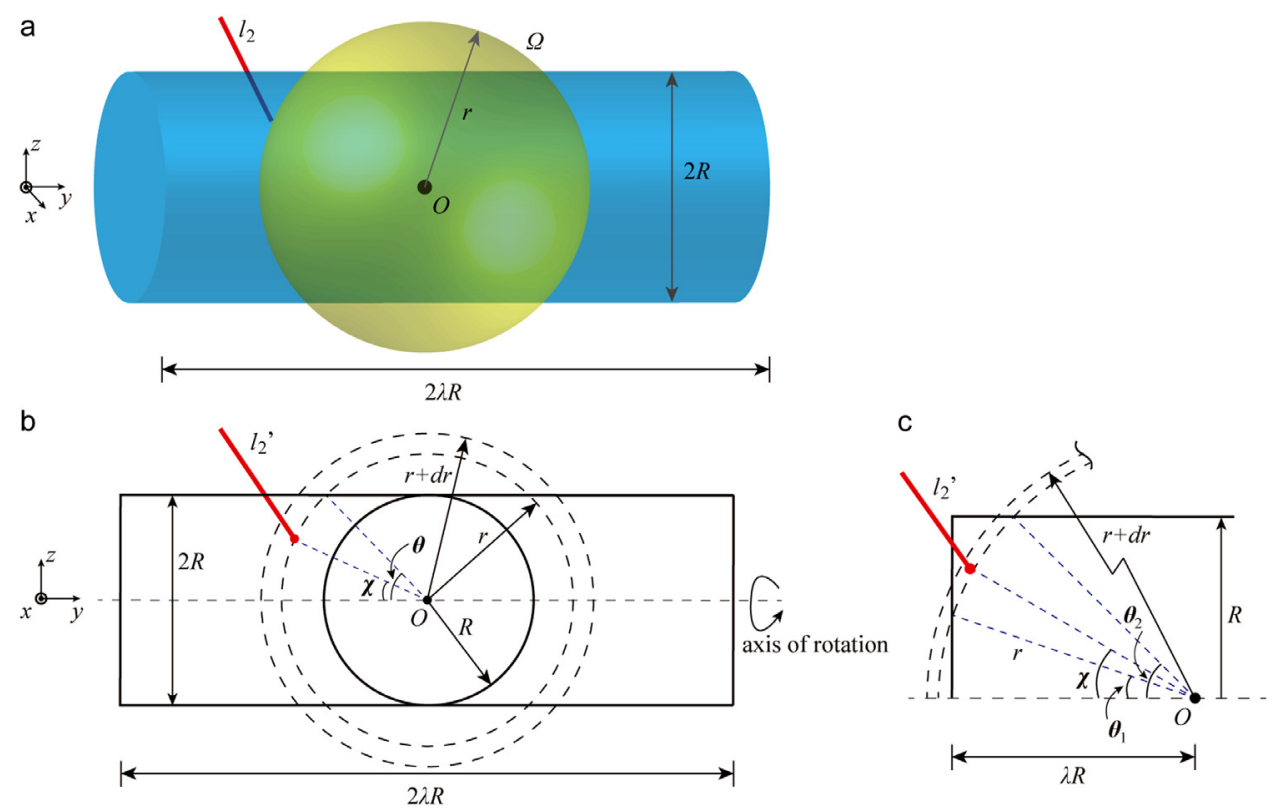

Fig. A1. Fibers in end contact with $\boldsymbol{\Omega}$ with contact points within a cylindrical NP. (a) Schematic showing a fiber $\left(l_{2}\right)$ in end contact with $\boldsymbol{\Omega}$ with contact point within the cylinder. (b) and (c) Projection of the cylinder and the fiber $\left(l_{2}{ }^{\prime}\right)$ on the $y z$-plane. $r$, the radius of $\boldsymbol{\Omega}$, is in the range of (b) $[R, \lambda R]$ and (c) $\left[\lambda R, \sqrt{\lambda^{2}+1} R\right]$.

within the region $[0, R]$ is $\gamma_{e} \cdot R^{3}$ where $\gamma_{e}=\frac{4 \pi v}{3}$; the number whose contact points fall within the region $[r, r+d r]$ is $\gamma_{e} \cdot d\left(r^{3}\right)$. As shown in Fig. A1, the number of fibers whose contact points fall within $[r, r+d r]$ and the cylinder is (these fibers are all in contact with the cylinder)

$$
\left\{\begin{array}{l}
\gamma_{e} \cdot d\left(r^{3}\right), r \in[0, R] \\
\gamma_{e} \cdot d\left(r^{3}\right) \cdot(1-\cos \theta), r \in[R, \lambda R] \\
\gamma_{e} \cdot d\left(r^{3}\right) \cdot\left(\cos \theta_{1}-\cos \theta_{2}\right), r \in\left[\lambda R, \sqrt{\lambda^{2}+1} R\right]
\end{array}\right.
$$

where $\cos \theta=\frac{\sqrt{r^{2}-R^{2}}}{r}, \cos \theta_{1}=\frac{\lambda R}{r}, \cos \theta_{2}=\frac{\sqrt{r^{2}-R^{2}}}{r}$.

Therefore, $\bar{N}_{2}$ is given by

$$
\begin{aligned}
\bar{N}_{2} & =\gamma_{e}\left[R^{3}+\int_{R}^{\lambda R}(1-\cos \theta) \cdot d r^{3}+\int_{\lambda R}^{\sqrt{\lambda^{2}+1} R}\left(\cos \theta_{1}-\cos \theta_{2}\right) \cdot d r^{3}\right] \\
& =\gamma_{e} \cdot g(\lambda) \cdot R^{3}
\end{aligned}
$$

where $g(\lambda)=\frac{3 \lambda}{2}$.

\section{A.2. Fibers in end contact with $\Omega$ with contact points outside the NP}

Next we deduce $\bar{N}_{4}$, the average number of fibers having end contact with $\Omega$ with contact points outside the NP $\left(l_{4}\right.$, Fig. 4(b)). These fibers can be classified into two kinds $\left(l_{4 a}\right.$ and $\left.l_{4 b}\right)$ : the contact points are located outside the side of the cylinder (region I in Fig. A2(b)), and the contact points are located outside the bottom side of the cylinder (region II in Fig. A2(b)). The number of these two kinds of fibers is denoted by $\bar{N}_{4 a}$ and $\bar{N}_{4 b}$, respectively. Then

$$
\bar{N}_{4}=\bar{N}_{4 a}+\bar{N}_{4 b} \text {. }
$$

(1) For fibers in end contact with $\boldsymbol{\Omega}$ with contact points within the region I (Fig. A2(c)), $\alpha_{2}$ should be in the range of $\left[\alpha_{2 \min }, \alpha_{2 \max }\right.$, where $\alpha_{2 \min }=\theta-\alpha_{1}$ with $\alpha_{1}=\operatorname{atan} \frac{1}{\lambda}$ and $\theta=\operatorname{asin} \frac{R}{r} . \alpha_{2 \max }$ is related to the length of the fiber and given by

$$
\alpha_{2 \max }=\left\{\begin{array}{l}
\operatorname{acos} \frac{r}{\sqrt{1+\lambda^{2}} R}, 2 L \geq \sqrt{\left(1+\lambda^{2}\right) R^{2}-r^{2}} \\
\operatorname{asin}\left[\frac{R r+2 L \cdot \sqrt{4 L^{2}+\left(r^{2}-R^{2}\right)}}{r^{2}+4 L^{2}}\right]-\alpha_{1}, \quad 2 L<\sqrt{\left(1+\lambda^{2}\right) R^{2}-r^{2}} .
\end{array}\right.
$$



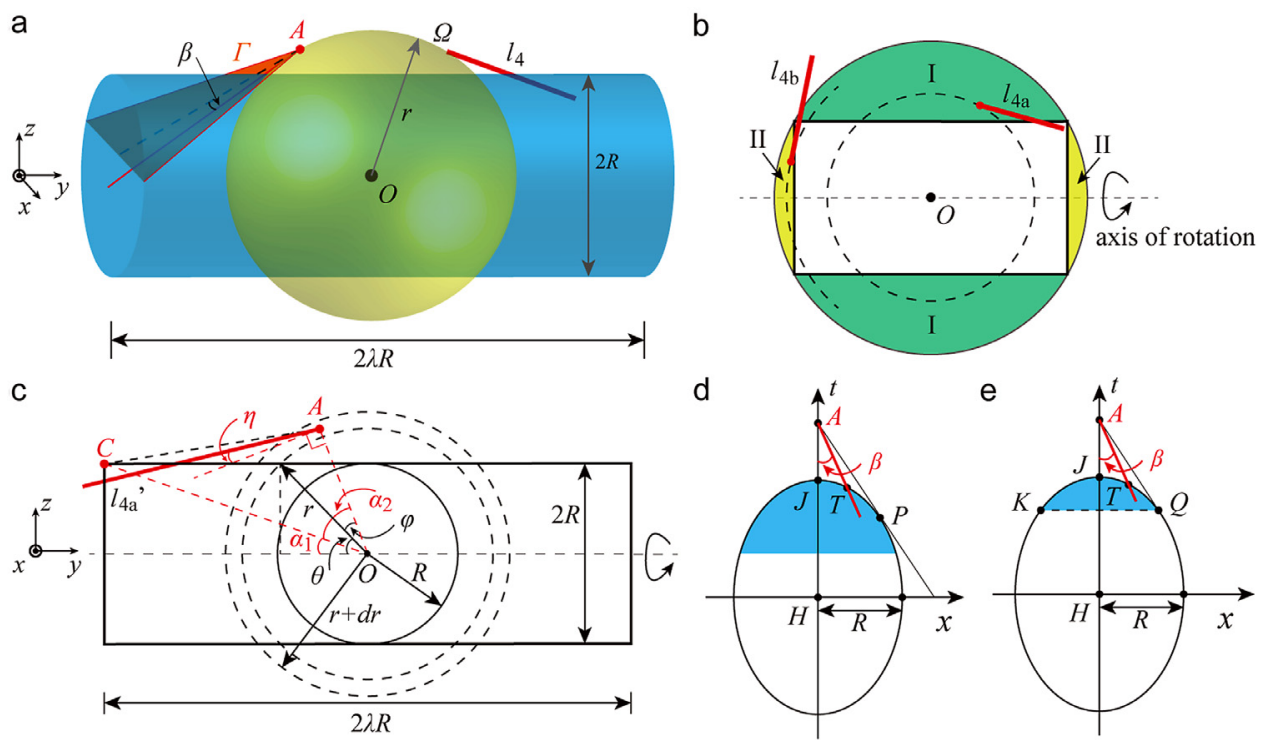

Fig. A2. Fibers in end contact with $\boldsymbol{\Omega}$ with contact points outside the NPs. (a) Schematic showing fibers in end contact with $\boldsymbol{\Omega}\left(l_{4}\right)$ with contact points outside the cylinder. (b) These fibers $\left(l_{4}\right)$ are classified into two kinds $\left(l_{4 a}\right.$ and $\left.l_{4 b}\right)$ : the contact points located outside the side of the cylinder (region I), and located outside the bottom side of the cylinder (region II). (c) Projection of the cylinder and the fiber $\left(l_{4 a}{ }^{\prime}\right)$ on the $y z$-plane. $r$, the radius of $\boldsymbol{\Omega}$, is in the range of $\left[R, \sqrt{\lambda^{2}+1} R\right]$. (d) and (e) Intersection of the $\Gamma$-plane with the cylinder or the extension of the cylinder, and the blue part is the intersection of the $\Gamma$-plane with the cylinder. (For interpretation of the references to color in this figure legend, the reader is referred to the web version of this article.)

In addition, for fibers with contact point $A$ and on $\Gamma$-plane (Fig. A2(a)), projection of these fibers on the $y z$-plane is $l_{4 a}{ }^{\prime}$ (Fig. A2(c)), and $\eta$ should be in the range of $\left[0, \eta_{\max }\right]$, where $\eta_{\max }$ is a function of $\alpha_{2}$ and the fiber length, $2 L$. $\eta_{\max }$ is given by

$$
\eta_{\max }=\left\{\begin{array}{l}
\operatorname{atan}\left[\frac{\sqrt{1+\lambda^{2}} R-r \cdot \cos \alpha_{2}}{r \cdot \sin \alpha_{2}}\right]-\alpha_{2}, 2 L \geq|A C| \\
\operatorname{acos}\left[\frac{r \cdot \sin \left(\alpha_{1}+\alpha_{2}\right)-R}{2 L}\right]-\left(\alpha_{1}+\alpha_{2}\right), 2 L<|A C|
\end{array},\right.
$$

where $|A C|$ is the distance between point $\mathrm{A}$ and point $\mathrm{C}$ (Fig. $\mathrm{A} 2(\mathrm{c})$ ) and given by $|A C|=$ $\sqrt{\left(1+\lambda^{2}\right) R^{2}+r^{2}-2 \sqrt{1+\lambda^{2}} R \cdot r \cdot \cos \alpha_{2}}$. Meanwhile, for fibers with the contact point $\mathrm{A}$ and on the $\Gamma$-plane, when the fibers are in contact with the cylinder, $\beta$ should be in the range of $\left[0, \beta_{\max }\right]$. $\beta_{\max }$ is a function of $\alpha_{2}, \eta$ and $L$. Next we will obtain the expression of $\beta_{\max }$.

Intersection of the $\Gamma$-plane with the cylinder or the extension of the cylinder is an ellipse, as shown in Figs. A2(d) and (e), and the blue part is the intersection of the $\Gamma$-plane with the cylinder. Point $P$, which is the tangent point where line segment AP intersects the ellipse, may be on the cylinder (Fig. A2(d)) or on the extension of the cylinder (Fig. A2 (e)). The coordinate components of point $P\left(x_{P}, y_{P}, z_{P}, t_{P}\right)$ are given by

$$
\left\{\begin{array}{l}
x_{P}=\frac{R}{r \cdot \sin \left(\alpha_{1}+\alpha_{2}\right)} \sqrt{r^{2} \cdot \sin ^{2}\left(\alpha_{1}+\alpha_{2}\right)-R^{2}} \\
y_{P}=-\left[\frac{r^{2} \cdot \sin ^{2}\left(\alpha_{1}+\alpha_{2}\right)-R^{2}}{r \cdot \sin \left(\alpha_{1}+\alpha_{2}\right) \cdot \cos \left(\alpha_{1}+\alpha_{2}+\eta\right)} \cdot \sin \left(\alpha_{1}+\alpha_{2}+\eta\right)+r \cdot \cos \left(\alpha_{1}+\alpha_{2}\right)\right] . \\
z_{P}=\frac{R^{2}}{r \cdot \sin \left(\alpha_{1}+\alpha_{2}\right)} \\
t_{P}=\frac{R^{2}}{r \cdot \sin \left(\alpha_{1}+\alpha_{2}\right) \cdot \cos \left(\alpha_{1}+\alpha_{2}+\eta\right)}
\end{array}\right.
$$

(i) When point $\mathrm{P}$ is on the cylinder, the inequality $y_{P} \geq-\lambda R$ must be satisfied. The inequality $y_{P} \geq-\lambda R$ is equivalent to the following inequality:

$$
\alpha_{2} \leq \operatorname{acos}\left(\frac{r^{2}-R^{2}}{\lambda \cdot R \cdot r}\right)-\alpha_{1}=\alpha_{C}
$$

and

$$
\eta \leq \operatorname{atan}\left\{\frac{\left[\lambda R-r \cdot \cos \left(\alpha_{1}+\alpha_{2}\right)\right] \cdot r \cdot \sin \left(\alpha_{1}+\alpha_{2}\right)}{r^{2} \cdot \sin ^{2}\left(\alpha_{1}+\alpha_{2}\right)-R^{2}}\right\}-\left(\alpha_{1}+\alpha_{2}\right)=\eta_{C}
$$


The length of segment AP is:

$$
|A P|=\sqrt{\frac{\left[r^{2} \cdot \sin ^{2}\left(\alpha_{1}+\alpha_{2}\right)-R^{2}\right] \cdot\left[r^{2} \cdot \sin ^{2}\left(\alpha_{1}+\alpha_{2}\right)-R^{2} \cdot \sin ^{2}\left(\alpha_{1}+\alpha_{2}+\eta\right)\right]}{r^{2} \cdot \sin ^{2}\left(\alpha_{1}+\alpha_{2}\right) \cdot \cos ^{2}\left(\alpha_{1}+\alpha_{2}+\eta\right)}} .
$$

When

$$
|A P| \leq 2 L,
$$

$\beta_{\max }=\angle \mathrm{PAH}=\beta_{1}$ (Fig. A2(d)). $\beta_{1}$ is given by

$$
\beta_{1}=\operatorname{acot}\left[\frac{\sqrt{r^{2} \cdot \sin ^{2}\left(\alpha_{1}+\alpha_{2}\right)-R^{2}}}{R \cdot \cos \left(\alpha_{1}+\alpha_{2}+\eta\right)}\right] \text {. }
$$

When

$$
|A P|>2 L,
$$

$\beta_{\max }=\angle \mathrm{JAT}=\beta_{2}$ with $|A T|=2 L$ (Fig. A2(d)). $\beta_{2}$ is given by

$$
\beta_{2}=\operatorname{acos} \frac{-r \cdot \sin \left(\alpha_{1}+\alpha_{2}\right) \cdot \cos \left(\alpha_{1}+\alpha_{2}+\eta\right)+\sqrt{r^{2} \cdot \sin ^{2}\left(\alpha_{1}+\alpha_{2}\right)+\left(4 L^{2}-R^{2}\right) \cdot \sin ^{2}\left(\alpha_{1}+\alpha_{2}+\eta\right)}}{2 L \cdot \sin ^{2}\left(\alpha_{1}+\alpha_{2}+\eta\right)} .
$$
$\beta_{2}$.

In short, when Eqs. (A7a), (A7b) and (A9) are satisfied, $\beta_{\max }=\beta_{1}$; when Eqs. (A7a), (A7b) and (A11) are satisfied, $\beta_{\max }=$

(ii) When point $\mathrm{P}$ is on the extension of the cylinder, then $y_{P}<-\lambda R$, which means Eqs. (A7a) and (A7b) are not all satisfied. In this case, the line KQ is the intersection of $\Gamma$-plane and the bottom of the cylinder (Fig. A2(e)). The coordinate components of point $Q\left(x_{Q}, y_{Q}, z_{Q}, t_{Q}\right)$ are given by

$$
\left\{\begin{array}{l}
x_{Q}=\sqrt{R^{2}-\left[r \cdot \sin \left(\alpha_{1}+\alpha_{2}\right)-\frac{\lambda R-r \cdot \cos \left(\alpha_{1}+\alpha_{2}\right)}{\tan \left(\alpha_{1}+\alpha_{2}+\eta\right)}\right]^{2}} \\
y_{Q}=-\lambda R \\
z_{Q}=r \cdot \sin \left(\alpha_{1}+\alpha_{2}\right)-\frac{\lambda R-r \cdot \cos \left(\alpha_{1}+\alpha_{2}\right)}{\tan \left(\alpha_{1}+\alpha_{2}+\eta\right)} \\
t_{Q}=\frac{r \cdot \sin \left(\alpha_{1}+\alpha_{2}\right)}{\cos \left(\alpha_{1}+\alpha_{2}+\eta\right)}-\frac{\lambda R-r \cdot \cos \left(\alpha_{1}+\alpha_{2}\right)}{\sin \left(\alpha_{1}+\alpha_{2}+\eta\right)}
\end{array} .\right.
$$

The length of the segment $A Q$ is

$$
|A Q|=\left\{R^{2}-r^{2} \cdot \sin ^{2}\left(\alpha_{1}+\alpha_{2}\right)+\left[\lambda R-r \cdot \cos \left(\alpha_{1}+\alpha_{2}\right)\right]^{2}+2 r \cdot \sin \left(\alpha_{1}+\alpha_{2}\right) \cdot \frac{\lambda R-r \cdot \cos \left(\alpha_{1}+\alpha_{2}\right)}{\tan \left(\alpha_{1}+\alpha_{2}+\eta\right)}\right\}^{1 / 2} .
$$

When

$$
|A Q| \leq 2 L,
$$

$\beta_{\max }=\angle \mathrm{QAH}=\beta_{3}$ (Fig. A2(e)). $\beta_{3}$ is given by

$$
\beta_{3}=\operatorname{atan} \frac{\sqrt{\left[R \cdot \sin \left(\alpha_{1}+\alpha_{2}+\eta\right)\right]^{2}-\left\{r \cdot \sin \left(\alpha_{1}+\alpha_{2}\right) \cdot \sin \left(\alpha_{1}+\alpha_{2}+\eta\right)-\left[\lambda R-r \cdot \cos \left(\alpha_{1}+\alpha_{2}\right)\right] \cdot \cos \left(\alpha_{1}+\alpha_{2}+\eta\right)\right\}^{2}}}{\lambda R-r \cdot \cos \left(\alpha_{1}+\alpha_{2}\right)} .
$$

When

$$
|A Q|>2 L,
$$

$\beta_{\max }=\beta_{2}$, and $\beta_{2}$ is given by Eq. (A12).

For all fibers in end contact with $\boldsymbol{\Omega}$ at point $\mathrm{A}$, the proportion of fibers in contact with the cylinder is

$$
\int_{0}^{\eta_{\max }} \frac{2 L^{2} \cdot \sin \beta}{2 \pi L^{2}} d \eta=\int_{0}^{\eta_{\max }} \frac{\sin \beta}{\pi} d \eta
$$

The number of fibers having end contact with $\boldsymbol{\Omega}$ in the region $[r, r+d r] \cap\left[\alpha_{2}, \alpha_{2}+d \alpha_{2}\right]$ is $\gamma_{e} \cdot d\left(r^{3}\right) \cdot \sin \left(\alpha_{1}+\alpha_{2}\right) \cdot d \alpha_{2}$, and the number of fibers having contact with the cylinder in these fibers is

$$
\gamma_{e} \cdot \sin \left(\alpha_{1}+\alpha_{2}\right) \cdot \int_{0}^{\eta_{\max }} \frac{\sin \beta}{\pi} d \eta \cdot d \alpha_{2} \cdot d\left(r^{3}\right)
$$




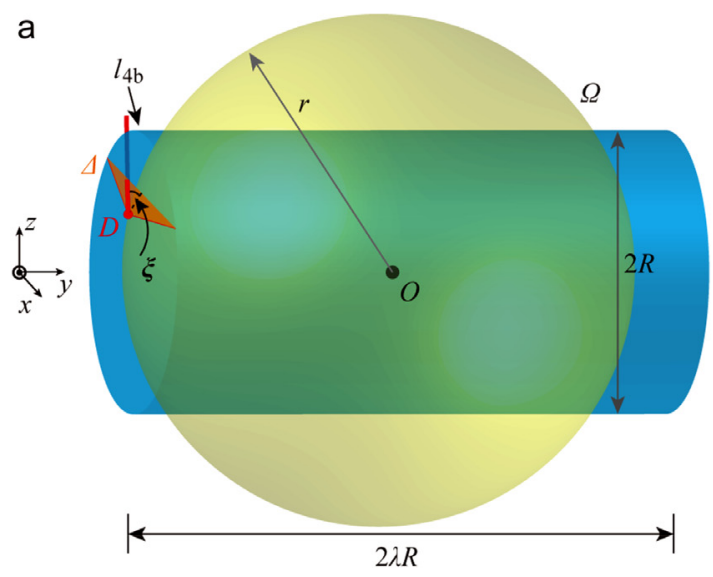

C

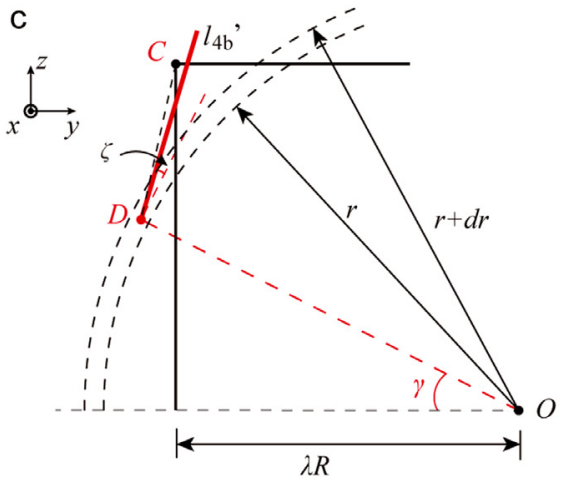

b

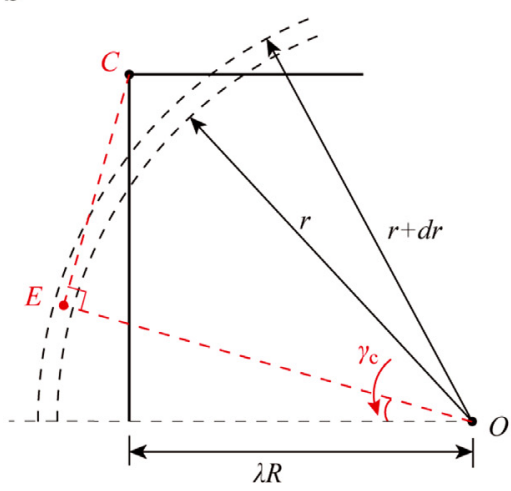

d

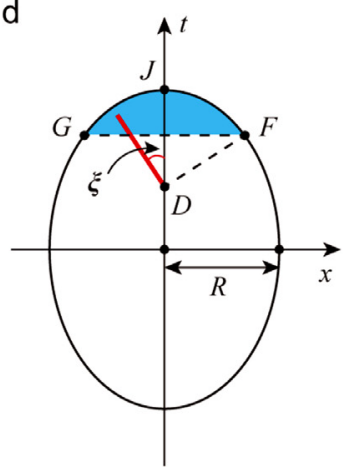

e

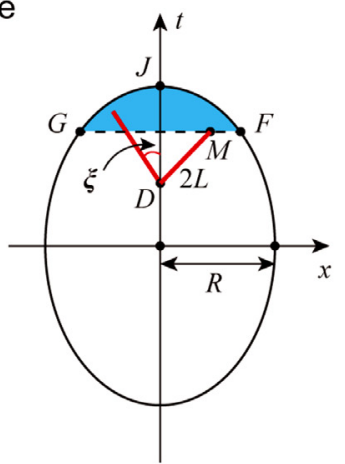

Fig. A3. Fibers in end contact with $\boldsymbol{\Omega}$ with contact points outside the NP. (a) Schematic showing a fiber in end contact with $\boldsymbol{\Omega}$ ( $l_{4 b}$ ) with contact point outside the bottom side of the cylinder. (b) Projection of the cylinder on the $y z$-plane and illustration of $\gamma_{c}$. (c) Projection of the cylinder and the fiber $\left(l_{4 b}{ }^{\prime}\right)$ on the $y z$-plane. $r$, the radius of $\Omega$, is in the range of $\left[\lambda R, \sqrt{\lambda^{2}+1} R\right]$. (d) and (e) Intersection of the $\Delta$-plane with the cylinder (the area in blue). (For interpretation of the references to color in this figure legend, the reader is referred to the web version of this article.)

Integrating Eq. (A19) with variables $\alpha_{2}$ (from $\alpha_{2 \min }$ to $\alpha_{2 \max }$ ) and $r$ (from $R$ to $\sqrt{1+\lambda^{2}} R$ ), we obtain $\bar{N}_{4 a}$, which is expressed as

$$
\begin{aligned}
\bar{N}_{4 a} & =\gamma_{e} \cdot \int_{R}^{\sqrt{1+\lambda^{2}} R}\left[\int_{\alpha_{2 \min }}^{\alpha_{2 \max }} \sin \left(\alpha_{1}+\alpha_{2}\right) \cdot \int_{0}^{\eta_{\max }} \frac{\sin \beta}{\pi} d \eta \cdot d \alpha_{2}\right] \cdot d\left(r^{3}\right) \\
& =\gamma_{e} \cdot H_{1}(\lambda, R, L),
\end{aligned}
$$

where $H_{1}(\lambda, R, L)=\int_{R}^{\sqrt{1+\lambda^{2}} R}\left[\int_{\alpha_{2 \min }}^{\alpha_{2 \max }} \sin \left(\alpha_{1}+\alpha_{2}\right) \cdot \int_{0}^{\eta_{\max }} \frac{\sin \beta}{\pi} d \eta \cdot d \alpha_{2}\right] \cdot d\left(r^{3}\right)$.

(2) For fibers in end contact with $\boldsymbol{\Omega}$ with contact points within the region II (Fig. A2(b)), $\gamma$ should be in the range of $\left[\gamma_{\min }, \gamma_{\max }\right]$ (Fig. A3(c)), where $\gamma_{\max }=\operatorname{acos} \frac{\lambda R}{r}$,

$$
\gamma_{\text {min }}=\left\{\begin{array}{l}
\operatorname{asin}\left[\frac{r-\sqrt{r^{2}-\left(1+\lambda^{2}\right)\left(r^{2}-\lambda^{2} R^{2}\right)}}{\left(1+\lambda^{2}\right)^{R}}\right], 2 L \geq|C E| \\
\operatorname{asin}\left[\frac{\sqrt{4 L^{2}+r^{2}-\lambda^{2} R^{2}} \cdot r-2 L \cdot \lambda R}{4 L^{2}+r^{2}}\right], 2 L<|C E|
\end{array}, \text { with }|C E|=\frac{R-r \cdot \sin \gamma_{c}}{\cos \gamma_{c}}\right.
$$

and $\gamma_{c}=\operatorname{asin} \frac{r-\sqrt{r^{2}-\left(1+\lambda^{2}\right)\left(r^{2}-\lambda^{2} R^{2}\right)}}{\left(1+\lambda^{2}\right) R}$ (Fig. A3(b)).

In addition, for fibers with contact point $D$ and on $\Delta$-plane (Fig. A3(a)), projection of these fibers on the $y z$-plane is $l_{4 b}{ }^{\prime}$ (Fig. A3(c)), and $\zeta$ should be in the range of $\left[0, \zeta_{\max }\right]$, where $\zeta_{\max }$ is a function of $\gamma$ and the fiber length, $2 L$. $\zeta_{\max }$ is given by

$$
\zeta_{\max }=\left\{\begin{array}{l}
\gamma-\operatorname{atan}\left[\frac{r \cdot \cos \gamma-\lambda R}{R-r \cdot \sin \gamma}\right], \quad 2 L \geq|C D| \\
\gamma-\operatorname{asin} \frac{r \cdot \cos \gamma-\lambda R}{2 L}, 2 L<|C D|
\end{array},\right.
$$


where $|C D|$ is the distance between point $C$ and point $\mathrm{D}$ (Fig. $\mathrm{A} 3(\mathrm{c}))$ given by $|C D|=\sqrt{(r \cdot \cos \gamma-\lambda R)^{2}+(R-r \cdot \sin \gamma)^{2}}$. Meanwhile, for fibers with the contact point $D$ and on the $\Delta$-plane, when the fibers are in contact with the cylinder, $\xi$ should be in the range of $\left[0, \xi_{\max }\right] . \xi_{\max }$ is a function of $\gamma, \zeta$ and $L$. Next we will deduce the expression of $\xi_{\text {max }}$.

Intersection of the $\Delta$-plane with the cylinder is the blue part of the ellipse (Fig. A3(d) and (e)). The coordinate components of point $F\left(x_{F}, y_{F}, z_{F}, t_{F}\right)$ are given by

$$
\left\{\begin{array}{l}
x_{F}=\sqrt{R^{2}-\left[\frac{r \cdot \cos \zeta-\lambda R \cdot \cos (\gamma-\zeta)}{\sin (\gamma-\zeta)}\right]^{2}} \\
y_{F}=-\lambda R \\
z_{F}=\frac{r \cdot \cos \zeta-\lambda R \cdot \cos (\gamma-\zeta)}{\sin (\gamma-\zeta)} \\
t_{F}=\frac{r \cdot \sin \gamma}{\cos (\gamma-\zeta)}+\frac{r \cdot \cos \gamma-\lambda R}{\sin (\gamma-\zeta)}
\end{array} .\right.
$$

The length of segment DF is

$$
|D F|=\sqrt{\left(1+\lambda^{2}\right) R^{2}+2 \lambda R \cdot r \cdot \frac{\sin \zeta}{\sin (\gamma-\zeta)}-r^{2} \cdot \frac{\sin (\gamma+\zeta)}{\sin (\gamma-\zeta)}} .
$$

When

$$
|D F| \leq 2 L
$$

$\xi_{\max }=\angle J D F=\xi_{1}$ (Fig. A3(d)). $\xi_{1}$ is given by

$$
\xi_{1}=\operatorname{atan} \frac{\sqrt{[R \cdot \sin (\gamma-\zeta)]^{2}-[r \cdot \cos \zeta-\lambda R \cdot \cos (\gamma-\zeta)]^{2}}}{r \cdot \cos \gamma-\lambda R} .
$$

When

$$
|D F|>2 L
$$

$\xi_{\max }=\angle J D M=\xi_{2}$ with $|D M|=2 L$ (Fig. A3(e)). $\xi_{2}$ is given by

$$
\xi_{2}=\operatorname{acos} \frac{r \cdot \cos \gamma-\lambda R}{2 L \cdot \sin (\gamma-\zeta)}
$$

For all fibers having end contact with $\boldsymbol{\Omega}$ at point $\mathrm{D}$, the proportion of fibers in contact with the cylinder is

$$
\int_{0}^{\zeta_{\max }} \frac{2 L^{2} \cdot \sin \xi}{2 \pi L^{2}} d \zeta=\int_{0}^{\zeta_{\max }} \frac{\sin \xi}{\pi} d \zeta
$$

The number of fibers having end contact with $\Omega$ in the region $[r, r+d r] \cap[\gamma, \gamma+d \gamma]$ is $\gamma_{e} \cdot d\left(r^{3}\right) \cdot \sin \gamma \cdot d \gamma$, and the number of fibers having contact with the cylinder in these fibers is

$$
\gamma_{e} \cdot\left(\sin \gamma \cdot \int_{0}^{\zeta_{\max }} \frac{\sin \xi}{\pi} d \zeta \cdot d \gamma\right) \cdot d\left(r^{3}\right)
$$
as

Integrating Eq. (A29) with variables $\gamma$ (from $\gamma_{\min }$ to $\gamma_{\max }$ ) and $r$ (from $\lambda R$ to $\sqrt{1+\lambda^{2}} R$ ), we obtain $\bar{N}_{4 b}$, which is express

$$
\begin{aligned}
\bar{N}_{4 b} & =\gamma_{e} \cdot \int_{\lambda R}^{\sqrt{1+\lambda^{2}} R}\left[\int_{\gamma_{\min }}^{\gamma_{\max }}\left(\sin \gamma \cdot \int_{0}^{\zeta_{\max }} \frac{\sin \xi}{\pi} d \zeta\right) \cdot d \gamma\right] \cdot d r^{3} \\
& =\gamma_{e} \cdot H_{2}(\lambda, R, L),
\end{aligned}
$$

where $H_{2}(\lambda, R, L)=\int_{\lambda R}^{\sqrt{1+\lambda^{2}} R}\left[\int_{\gamma_{\min }}^{\gamma_{\max }}\left(\sin \gamma \cdot \int_{0}^{\zeta_{\max }} \frac{\sin \xi}{\pi} d \zeta\right) \cdot d \gamma\right] \cdot d r^{3}$.

\section{A.3. Fibers in tangential contact with $\Omega$ with contact points outside the NP}

Next we deduce $\bar{N}_{3}$, the average number of fibers having tangential contact with $\Omega$ with contact points outside the NP $\left(l_{3}\right.$, Fig. $\left.4(\mathrm{~b})\right)$. These fibers can also be classified into two kinds $\left(l_{3 a}\right.$ and $\left.l_{3 b}\right)$ : the contact points located outside the side of the cylinder (region I in Fig. A4(b)), and the contact points located outside the bottom side of the cylinder (region II in Fig. A4(b)). The number of these two kinds of fibers is $\bar{N}_{3 a}$ and $\bar{N}_{3 b}$, respectively. Then

$$
\bar{N}_{3}=\bar{N}_{3 a}+\bar{N}_{3 b} \text {. }
$$

(1) For fibers in tangential contact with $\boldsymbol{\Omega}$ with contact points within the region I (Fig. A4(c)), $\alpha_{2}$ should be in the range of $\left[\alpha_{2 \min }, \alpha_{2 \max }\right]$, where $\alpha_{2 \min }=\theta-\alpha_{1}$ with $\alpha_{1}=$ atan $\frac{1}{\lambda}$ and $\theta=\operatorname{asin} \frac{R}{r} . \alpha_{2 \max }$ is related to the length of the fiber and given 

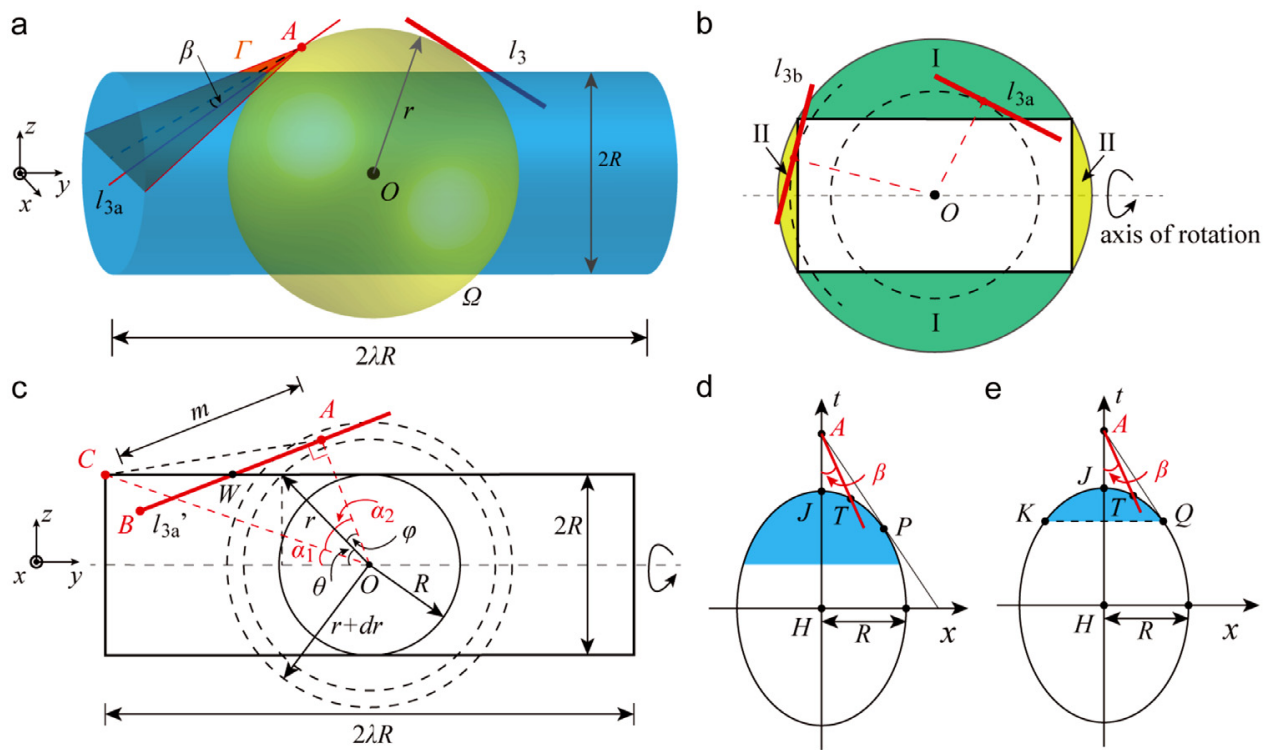

Fig. A4. Fibers in tangential contact with $\boldsymbol{\Omega}$ with contact points outside the NP. (a) Schematic showing fibers in contact with the cylinder $\left(l_{3}\right)$, which are in tangential contact with $\boldsymbol{\Omega}$ and the contact points are outside the cylinder. (b) These fibers $\left(l_{3}\right)$ are classified into two kinds $\left(l_{3 a}\right.$ and $\left.l_{3 b}\right)$ : the contact points located outside the side of the cylinder (region I), and the contact points located outside the bottom side of the cylinder (region II). (d) Projection of the cylinder and the fiber $\left(l_{3 a}{ }^{\prime}\right)$ on the $y z$-plane. $r$, the radius of $\boldsymbol{\Omega}$, is in the range of $\left[R, \sqrt{\lambda^{2}+1} R\right]$. (d) and (e) Intersection of the $\Gamma$-plane with the cylinder or the extension of the cylinder, and the blue part is the intersection of the $\Gamma$-plane with the cylinder. (For interpretation of the references to color in this figure legend, the reader is referred to the web version of this article.)

by Eq. (A4). For fibers with contact point $\mathrm{A}$ and on $\Gamma$-plane (Fig. A4(a)), projections of these fibers on the $y z$-plane is $l_{3 a}{ }^{\prime}$ (Fig. A4(c)). When the fibers are in contact with the cylinder, $m$ (the length of segment $A B$ ) should be in the range of $\left[m_{\min }\right.$, $2 L]$, where $m_{\min }$ is equal to the length of line segment AW (Fig. A4(c)) and given by

$$
m_{\min }=\frac{r \cdot \sin \left(\alpha_{1}+\alpha_{2}\right)-R}{\cos \left(\alpha_{1}+\alpha_{2}\right)} .
$$

Meanwhile, $\beta$ should be in the range of $\left[0, \beta_{\max }\right] . \beta_{\max }$ is a function of $\alpha_{2}, m$ and $L$. Next we will obtain the expression of $\beta_{\max }$.

Intersection of the $\Gamma$-plane with the cylinder or the extension of the cylinder is an ellipse, as shown in Fig. A4(d) and (e), and the blue part is the intersection of the $\Gamma$-plane with the cylinder. Point $P$, which is the tangent point where line segment AP intersects the ellipse, may be on the cylinder (Fig. A4(d)) or on the extension of the cylinder (Fig. A4(e)). The coordinate components of point $P\left(x_{P}, y_{P}, z_{P}, t_{P}\right)$ are given by

$$
\left\{\begin{array}{l}
x_{P}=\frac{R}{r \cdot \sin \left(\alpha_{1}+\alpha_{2}\right)} \sqrt{r^{2} \cdot \sin ^{2}\left(\alpha_{1}+\alpha_{2}\right)-R^{2}} \\
y_{P}=-\frac{r^{2}-R^{2}}{r \cdot \cos \left(\alpha_{1}+\alpha_{2}\right)} \\
z_{P}=\frac{R^{2}}{r \cdot \sin \left(\alpha_{1}+\alpha_{2}\right)} \\
t_{P}=\frac{R^{2}}{r \cdot \sin \left(\alpha_{1}+\alpha_{2}\right) \cdot \cos \left(\alpha_{1}+\alpha_{2}\right)}
\end{array} .\right.
$$

(i) When point $\mathrm{P}$ is on the cylinder, the inequality $y_{P} \geq-\lambda R$ must be satisfied, which is equivalent to the following inequality

$$
\alpha_{2} \leq \operatorname{acos}\left(\frac{r^{2}-R^{2}}{\lambda \cdot R \cdot r}\right)-\alpha_{1}=\alpha_{C} .
$$

The length of segment AP is given by

$$
|A P|=\sqrt{\frac{\left[r^{2} \cdot \sin ^{2}\left(\alpha_{1}+\alpha_{2}\right)-R^{2}\right] \cdot\left(r^{2}-R^{2}\right)}{r^{2} \cdot \cos ^{2}\left(\alpha_{1}+\alpha_{2}\right)}} .
$$

When

$$
|A P| \leq m,
$$


$\beta_{\max }=\angle \mathrm{PAH}=\beta_{4}$ (Fig. A4(d)). $\beta_{4}$ is given by

$$
\beta_{4}=\operatorname{acot}\left[\frac{\sqrt{r^{2} \cdot \sin ^{2}\left(\alpha_{1}+\alpha_{2}\right)-R^{2}}}{R \cdot \cos \left(\alpha_{1}+\alpha_{2}\right)}\right] \text {. }
$$

When

$$
|A P|>m,
$$

$\beta_{\max }=\angle \mathrm{JAT}=\beta_{5}$ with $|A T|=m$ (Fig. A4(d)). $\beta_{5}$ is given by

$$
\beta_{5}=\operatorname{acos} \frac{-r \cdot \cos \left(\alpha_{1}+\alpha_{2}\right)+\sqrt{r^{2}+\left(m^{2}-R^{2}\right)}}{m \cdot \sin \left(\alpha_{1}+\alpha_{2}\right)} .
$$

In short, when Eqs. (A34) and (A36) are satisfied, $\beta_{\max }=\beta_{4}$; when Eqs. (A34) and (A38) are satisfied, $\beta_{\max }=\beta_{5}$.

(ii) When point $\mathrm{P}$ is on the extension of the cylinder, then $y_{P}<-\lambda R$, which means Eq. (A34) is not satisfied. In this case, the line $\mathrm{KQ}$ is the intersection of $\Gamma$-plane and the bottom of the cylinder (Fig. A4(e)). The coordinate components of point $Q\left(x_{Q}, y_{Q}, z_{Q}, t_{Q}\right)$ are given by

$$
\left\{\begin{array}{l}
x_{Q}=\sqrt{R^{2}-\left[\frac{r-\lambda R \cdot \cos \left(\alpha_{1}+\alpha_{2}\right)}{\sin \left(\alpha_{1}+\alpha_{2}\right)}\right]^{2}} \\
y_{Q}=-\lambda R \\
z_{Q}=\frac{r-\lambda R \cdot \cos \left(\alpha_{1}+\alpha_{2}\right)}{\sin \left(\alpha_{1}+\alpha_{2}\right)} \\
t_{Q}=\frac{r-\lambda R \cdot \cos \left(\alpha_{1}+\alpha_{2}\right)}{\sin \left(\alpha_{1}+\alpha_{2}\right) \cdot \cos \left(\alpha_{1}+\alpha_{2}\right)}
\end{array} .\right.
$$

The length of segment $A Q$ is

$$
|A Q|=\sqrt{\left(1+\lambda^{2}\right) R^{2}-r^{2}}
$$

When

$$
|A Q| \leq m,
$$

$\beta_{\max }=\angle \mathrm{QAH}=\beta_{6}$ (Fig. A4(e)). $\beta_{6}$ is given by

$$
\beta_{6}=\operatorname{atan} \frac{\sqrt{R^{2}-r^{2}-\left(1+\lambda^{2}\right) R^{2} \cdot \cos ^{2}\left(\alpha_{1}+\alpha_{2}\right)+2 \lambda \cdot R \cdot r \cdot \cos \left(\alpha_{1}+\alpha_{2}\right)}}{\lambda R-r \cdot \cos \left(\alpha_{1}+\alpha_{2}\right)} .
$$

When

$$
|A Q|>m,
$$

$\beta_{\max }=\beta_{5}$, and $\beta_{5}$ is given by Eq. (A39).

For all fibers in tangential contact with $\Omega$ at point $A$, the proportion of fibers having contact with the cylinder is

$$
\int_{m_{\min }}^{2 L} \frac{2 \beta}{\pi} \cdot \frac{d m}{2 L}
$$

The number of fibers having tangential contact with the spherical surface $\boldsymbol{\Omega}$ in the region $[r, r+d r] \cap\left[\alpha_{2}, \alpha_{2}+d \alpha_{2}\right]$ is $\gamma_{t} \cdot d\left(r^{2}\right) \cdot \sin \left(\alpha_{1}+\alpha_{2}\right) \cdot d \alpha_{2}$, and the number of fibers having contact with the cylinder in these fibers is

$$
\gamma_{t} \cdot \sin \left(\alpha_{1}+\alpha_{2}\right) \cdot \int_{m_{\min }}^{2 L} \frac{2 \beta}{\pi} \cdot \frac{d m}{2 L} \cdot d \alpha_{2} \cdot d r^{2}
$$

Integrating Eq. (A46) with variables $\alpha_{2}$ (from $\alpha_{2 \min }$ to $\alpha_{2 \max }$ ) and $r$ (from $R$ to $\sqrt{1+\lambda^{2}} R$ ), we obtain $\bar{N}_{3 a}$ expressed as

$$
\begin{aligned}
\bar{N}_{3 a} & =\gamma_{t} \cdot \int_{R}^{\sqrt{1+\lambda^{2}} R}\left[\int_{\alpha_{2 \min }}^{\alpha_{2 \max }} \sin \left(\alpha_{1}+\alpha_{2}\right) \cdot \int_{m_{\min }}^{2 L} \frac{2 \beta}{\pi} \cdot \frac{d m}{2 L} \cdot d \alpha_{2}\right] \cdot d r^{2} \\
& =\gamma_{t} \cdot G_{1}(\lambda, R, L),
\end{aligned}
$$

where $G_{1}(\lambda, R, L)=\int_{R}^{\sqrt{1+\lambda^{2}} R}\left[\int_{\alpha_{2 \min }}^{\alpha_{2 \max }} \sin \left(\alpha_{1}+\alpha_{2}\right) \cdot \int_{m_{\min }}^{2 L} \frac{2 \beta}{\pi} \cdot \frac{d m}{2 L} \cdot d \alpha_{2}\right] \cdot d r^{2}$. 


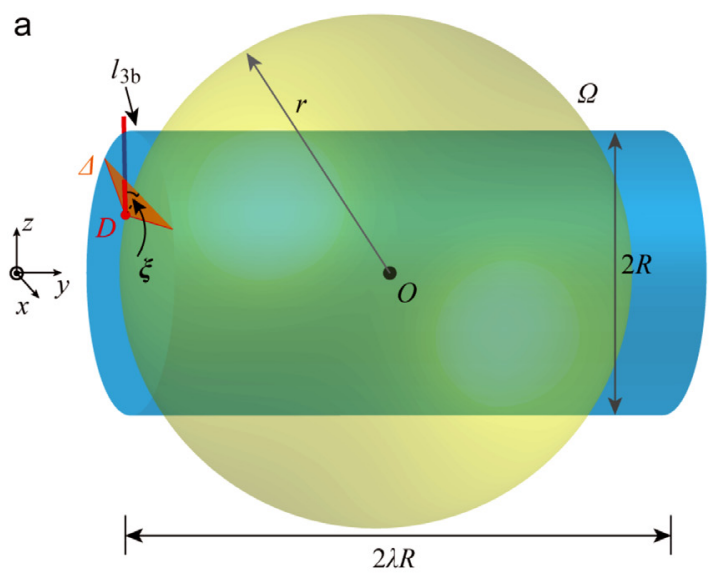

C

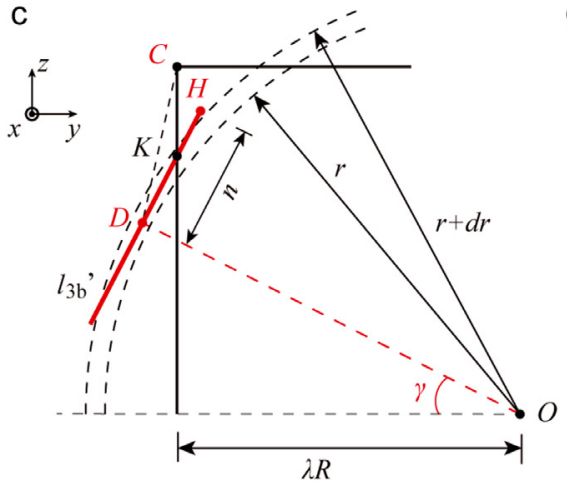

b

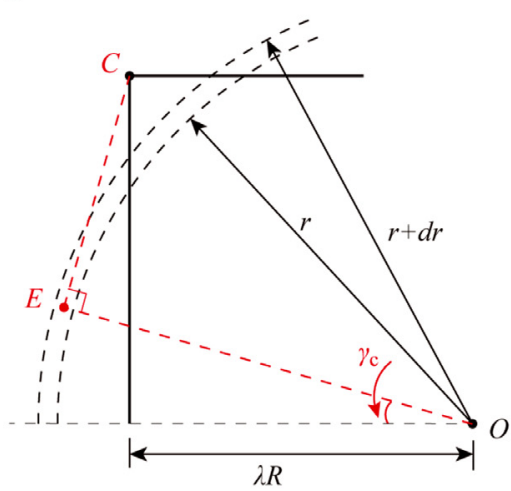

e d

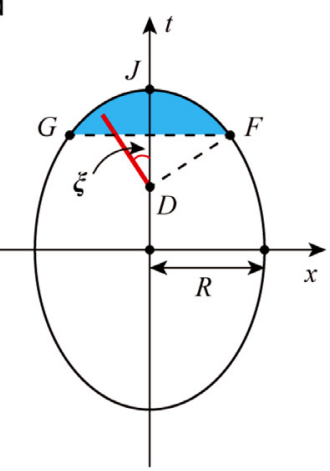

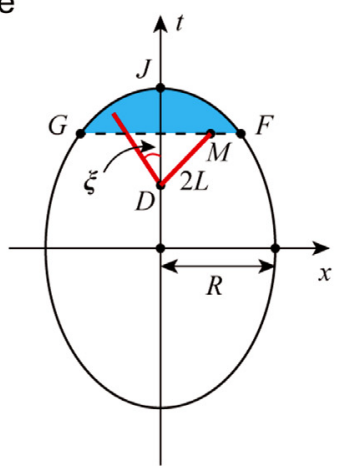

Fig. A5. Fibers in tangential contact with $\boldsymbol{\Omega}$ with contact points outside the NP. (a) Schematic showing a fiber in tangential contact with $\boldsymbol{\Omega}\left(l_{3 b}\right)$ with contact point outside the bottom side of the cylinder. (b) Projection of the cylinder on the $y z$-plane and illustration of $\gamma_{c}$. (c) Projection of the cylinder and the fiber $\left(l_{3 b^{\prime}}\right)$ on the $y z$-plane. $r$, the radius of $\boldsymbol{\Omega}$, is in the range of $\left[\lambda R, \sqrt{\lambda^{2}+1} R\right]$. (d) and (e) Intersection of the $\Delta$-plane with the cylinder (the area in blue). (For interpretation of the references to color in this figure legend, the reader is referred to the web version of this article.)

(2) For fibers in tangential contact with $\Omega$ with contact points within the region II (Fig. A4(b)), $\gamma$ should be in the range of $\left[\gamma_{\min }, \gamma_{\max }\right]$, where $\gamma_{\max }=\operatorname{acos} \frac{\lambda R}{r}$,

$$
\gamma_{\text {min }}=\left\{\begin{array}{l}
\operatorname{asin}\left[\frac{r-\sqrt{r^{2}-\left(1+\lambda^{2}\right)\left(r^{2}-\lambda^{2} R^{2}\right)}}{\left(1+\lambda^{2}\right) R}\right], 2 L \geq|C E| \\
\operatorname{asin}\left[\frac{\sqrt{4 L^{2}+r^{2}-\lambda^{2} R^{2}} \cdot r-2 L \cdot \lambda R}{4 L^{2}+r^{2}}\right], 2 L<|C E|
\end{array}, \text { with }|C E|=\frac{R-r \cdot \sin \gamma_{c}}{\cos \gamma_{c}}\right.
$$

and $\gamma_{c}=\operatorname{asin} \frac{r-\sqrt{r^{2}-\left(1+\lambda^{2}\right)\left(r^{2}-\lambda^{2} R^{2}\right)}}{\left(1+\lambda^{2}\right) R}$ (Fig. A5(b)). In addition, for fibers with contact point D and on $\Delta$-plane (Fig. A5(a)), projections of these fibers on the $y z$-plane is $l_{3 b^{\prime}}{ }^{\prime}$ Fig. A5(c)). When the fibers are in contact with the cylinder, $n$ (the length of segment $\mathrm{DH}$ ) should be in the range of $\left[n_{\min }, 2 L\right]$, where $n_{\min }$ is equal to the length of line segment DK and given by

$$
n_{\min }=\frac{r \cdot \cos \gamma-\lambda R}{\sin \gamma}
$$

Meanwhile, $\xi$ should be in the range of $\left[0, \xi_{\max }\right] . \xi_{\max }$ is a function of $\gamma, n$ and $L$. Next we will obtain the expression of $\xi_{\max }$. 
Intersection of the $\Delta$-plane with the cylinder is the blue part of the ellipse (Fig. A5(d)). The coordinate components of point $F\left(x_{F}, y_{F}, z_{F}, t_{F}\right)$ are given by

$$
\left\{\begin{array}{l}
x_{F}=\sqrt{R^{2}-\left(\frac{r-\lambda R \cdot \cos \gamma}{\sin \gamma}\right)^{2}} \\
y_{F}=-\lambda R \\
z_{F}=\frac{r-\lambda R \cdot \cos \gamma}{\sin \gamma} \\
t_{F}=\frac{r-\lambda R \cdot \cos \gamma}{\sin \gamma \cdot \cos \gamma}
\end{array}\right.
$$

The length of segment DF is

$$
|D F|=\sqrt{\left(1+\lambda^{2}\right) R^{2}-r^{2}}
$$

When

$$
|D F| \leq n
$$

$\xi_{\max }=\angle J D F=\xi_{3}$ (Fig. A5(d)). $\xi_{3}$ is given by

$$
\xi_{3}=\operatorname{atan} \frac{\sqrt{(R \cdot \sin \gamma)^{2}-(r-\lambda R \cdot \cos \gamma)^{2}}}{r \cdot \cos \gamma-\lambda R} .
$$

When

$$
|D F|>n
$$

$\xi_{\max }=\angle J D M=\xi_{4}$ with $|D M|=n$ (Fig. A5(e)). $\xi_{4}$ is given by

$$
\xi_{4}=\operatorname{acos} \frac{r \cdot \cos \gamma-\lambda R}{m \cdot \sin \gamma} .
$$

For all fibers having tangential contact with $\boldsymbol{\Omega}$ at point $\mathrm{D}$, the proportion of fibers having contact with the cylinder is

$$
\int_{n_{\min }}^{2 L} \frac{2 \xi}{\pi} \cdot \frac{d n}{2 L}
$$

The number of fibers having tangential contact with $\boldsymbol{\Omega}$ in the region $[r, r+d r] \cap[\gamma, \gamma+d \gamma]$ is $\gamma_{t} \cdot d\left(r^{2}\right) \cdot \sin \gamma \cdot d \gamma$ and the number of fibers having contact with the cylinder in these fibers is

$$
\gamma_{t} \cdot\left(\sin \gamma \cdot \int_{n_{\min }}^{2 L} \frac{2 \xi}{\pi} \cdot \frac{d n}{2 L} \cdot d \gamma\right) \cdot d r^{2}
$$

Integrating Eq. (A56) with variables $\gamma$ (from $\gamma_{\min }$ to $\gamma_{\max }$ ) and $r$ (from $\lambda R$ to $\sqrt{1+\lambda^{2}} R$ ), we obtain $\bar{N}_{3 b}$ expressed as

$$
\begin{aligned}
\bar{N}_{3 b} & =\gamma_{t} \cdot \int_{\lambda R}^{\sqrt{1+\lambda^{2}} R}\left[\int_{\gamma_{\min }}^{\gamma_{\max }}\left(\sin \gamma \cdot \int_{n_{\min }}^{2 L} \frac{2 \xi}{\pi} \cdot \frac{d n}{2 L}\right) \cdot d \gamma\right] \cdot d r^{2} \\
& =\gamma_{t} \cdot G_{2}(\lambda, R, L),
\end{aligned}
$$

where $G_{2}(\lambda, R, L)=\int_{\lambda R}^{\sqrt{1+\lambda^{2}} R}\left[\int_{\gamma_{\min }}^{\gamma_{\max }}\left(\sin \gamma \cdot \int_{n_{\min }}^{2 L} \frac{2 \xi}{\pi} \cdot \frac{d n}{2 L}\right) \cdot d \gamma\right] \cdot d r^{2}$.

Table A1

Molar ratios of chemicals used in the fabrication of MSNRs.

\begin{tabular}{ll}
\hline MSNRs & Molar ratio of $\mathrm{H}_{2} \mathrm{O}:$ CTAB: $\mathrm{NH}_{4} \mathrm{OH}:$ TEOS \\
\hline AR2 & 1000: $0.12: 7.5: 1.1$ \\
AR3 & 1000: $0.2: 7.5: 0.7$ \\
AR4 & 1000: $0.4: 10: 1.4$ \\
AR8 & 1000: $0.5: 7.5: 0.8$ \\
\hline
\end{tabular}




\section{Appendix B. Experimental details}

\section{B.1. Materials}

Experiment reagents. RITC (Sigma-Aldrich), 3-aminopropyltriethoxysilane (APTES) (Aladdin), Cetyltrimethylammonium bromide (CTAB), tetraethoxysilane (TEOS) and other chemicals were purchased from the Sinopharm Chemical Reagent Company. Centrifuge (Beckman Coulter Allegra 64R centrifuge, USA), lyophilizer (Leica EM CPD300, Leica, Germany), transmission electron microscope (SU82220, HITACHI, Japan), Zetasizer (Nano-ZS, Malvern instruments, United Kingdom), fluorescence inverted microscope (DMI 4000B, Leica, Germany).

Animal care. Male Sprague-Dawley (SD) rats (6-8 weeks old) were provided by the Animal Experiments Center of the Shanghai Institute of Materia Medica, Shanghai, China. Animals had free access to rat chow and tap water. Animal experiments were carried out according to the Institutional Animal Care and Use Committee (IACUC) guidelines of the Shanghai Institute of Materia Medica. (IACUC code, 2015-12-GY-20)

\section{B.2. Methods}

Fabrication of MSNs. For AR1, $\mathrm{NaOH}(0.28 \mathrm{~g})$ and $\mathrm{CTAB}(1 \mathrm{~g})$ were dissolved in deionized water at $80{ }^{\circ} \mathrm{C}$ via vigorous stirring. TEOS $(5 \mathrm{ml})$ was then introduced dropwise, followed by continuous stirring for $2 \mathrm{~h}$. The solution was centrifuged and washed with ethanol three times, and the particles were resuspended in ethanol with $\mathrm{HCl}$ followed by overnight reaction to remove the CTAB. The final products were collected by lyophilization. For the MSNRs, CTAB and $\mathrm{NH}_{4} \mathrm{OH}$ were first dissolved in deionized water and stirred for $1 \mathrm{~h}$. After introducing TEOS dropwise, the reaction was allowed to take place for $3 \mathrm{~h}$ at room temperature. The following steps were the same as those of AR1. Molar ratios of each chemical was presented in Table A1.

RITC labeling. RITC was covalently bound with APTES in ethanol, and then the prepared MSNs were added to form the conjugation. (To control the similar zeta potentials of all particles, the molar ratio of APTES was 8:4:4:3:2 for AR1, AR2, AR3, AR4 and AR8, respectively.) The reaction was carried out for $8 \mathrm{~h}$, and the solution was centrifuged, washed with ethanol, and ultra-sonicated three times to remove the unconjugated RITC and APTES. Particles were collected and stored.

Characterization. Non-labeled MSNs were dissolved in ethanol, and the morphological images were captured by SEM. The hydrodynamic diameter and zeta potentials of the RITC-labeled particles were measured in Zeta sizer.

Multiple particle tracking. SD rats were fasted $18 \mathrm{~h}$ before sacrifice. Fresh mucus was carefully isolated from the inner surface of the intestinal lumen. Particle solution of $5 \mathrm{ul}(200 \mathrm{ug} / \mathrm{ml})$ was added to freshly obtained mucus (200 ul), followed by catching movies using The resolution was set to $32.6 \mathrm{~ms}$ in order to ensure enough frames caught per second for subsequent analysis. The trajectories and MSD at a time scale of $1 \mathrm{~s}$ were computed by ImageJ.

\section{Supplementary materials}

Supplementary material associated with this article can be found, in the online version, at doi:10.1016/j.jmps.2017.12.014.

\section{References}

Altenberger, A.R., Tirrell, M., Dahler, J.S., 1986. Hydrodynamic screening and particle dynamics in porous media, semidilute polymer solutions and polymer gels. J. Chem. Phys. 84, 5122-5130.

Amsden, B., 1998. Solute diffusion within hydrogels. Mechanisms and models. Macromolecules 31, 8382-8395.

Amsden, B., 1999. An obstruction-scaling model for diffusion in homogeneous hydrogels. Macromolecules 32, 874-879.

Button, B., Cai, L.H., Ehre, C., Kesimer, M., Hill, D.B., Sheehan, J.K., Boucher, R.C., Rubinstein, M., 2012. A periciliary brush promotes the lung health by separating the mucus layer from airway epithelia. Science 337, 937-941.

Cai, L.H., Panyukov, S., Rubinstein, M., 2011. Mobility of nonsticky nanoparticles in polymer liquids. Macromolecules 44, 7853-7863.

Cai, L.H., Panyukov, S., Rubinstein, M., 2015. Hopping diffusion of nanoparticles in polymer matrices. Macromolecules 48, $847-862$.

Chauhan, V.P., Popovic, Z., Chen, O., Cui, J., Fukumura, D., Bawendi, M.G., Jain, R.K., 2011. Fluorescent nanorods and nanospheres for real-time in vivo probing of nanoparticle shape-dependent tumor penetration. Angew. Chem. Int. Ed. 50, 11417-11420.

Choi, J., Cargnello, M., Murray, C.B., Clarke, N., Winey, K.I., Composto, R.J., 2015. Fast nanorod diffusion through entangled polymer melts. ACS Macro Lett 4, 952-956.

Chun, P.W., Thornby, J.I., Saw, J.G., 1969. Exclusion of protein from high polymer media .I. Derivation of probability distribution for the number of fiber centers within any sphere of radius r. Biophys. J. 9, 163-172.

Cone, R.A., 2009. Barrier properties of mucus. Adv. Drug Deliv. Rev. 61, 75-85

Cu, Y., Saltzman, W.M., 2009. Mathematical modeling of molecular diffusion through mucus. Adv. Drug Deliv. Rev. 61, 101-114.

Cukier, R.I., 1984. Diffusion of brownian spheres in semidilute polymer solutions. Macromolecules 17, $252-255$.

Fakhri, N., MacKintosh, F.C., Lounis, B., Cognet, L., Pasquali, M., 2010. Brownian motion of stiff filaments in a crowded environment. Science 330, $1804-1807$.

Geng, Y., Dalhaimer, P., Cai, S.S., Tsai, R., Tewari, M., Minko, T., Discher, D.E., 2007. Shape effects of filaments versus spherical particles in flow and drug delivery. Nat. Nanotechnol. 2, 249-255.

Hadjiev, N.A., Amsden, B.G., 2015. An assessment of the ability of the obstruction-scaling model to estimate solute diffusion coefficients in hydrogels. J. Control Release 199, 10-16.

Han, Y., Alsayed, A.M., Nobili, M., Zhang, J., Lubensky, T.C., Yodh, A.G., 2006. Brownian motion of an ellipsoid. Science 314, 626-630.

Hanggi, P., Talkner, P., Borkovec, M., 1990. Reaction-rate theory: fifty years after kramers. Rev. Mod. Phys. 62, 251-341.

Hinde, E., Thammasiraphop, K., Duong, H.T.T., Yeow, J., Karagoz, B., Boyer, C., Gooding, J.J., Gaus, K., 2017. Pair correlation microscopy reveals the role of nanoparticle shape in intracellular transport and site of drug release. Nat. Nanotechnol. 12, 81-89.

Hofling, F., Franosch, T., 2013. Anomalous transport in the crowded world of biological cells. Rep. Progr. Phys. 76, 046602.

Jain, R.K., Stylianopoulos, T., 2010. Delivering nanomedicine to solid tumors. Nat. Rev. Clin. Oncol. 7, 653-664. 
Johansson, L., Elvingson, C., Lofroth, J.E., 1991. Diffusion and interaction in gels and solutions. 3. Theoretical results on the obstruction effect. Macromolecules 24, 6024-6029.

Johansson, M.E.V., Sjovall, H., Hansson, G.C., 2013. The gastrointestinal mucus system in health and disease. Nat. Rev. Gastroenterol. Hepatol. 10, 352-361.

Kirch, J., Schneider, A., Abou, B., Hopf, A., Schaefer, U.F., Schneider, M., Schall, C., Wagner, C., Lehr, C.M., 2012. Optical tweezers reveal relationship between microstructure and nanoparticle penetration of pulmonary mucus. Proc. Natl. Acad. Sci. U. S. A. 109, 18355-18360.

Lai, S.K., O'Hanlon, D.E., Harrold, S., Man, S.T., Wang, Y.Y., Cone, R., Hanes, J., 2007. Rapid transport of large polymeric nanoparticles in fresh undiluted human mucus. In: Proc. Natl. Acad. Sci. U.S.A., 104, pp. 1482-1487.

Lai, S.K., Wang, Y.Y., Hanes, J., 2009. Mucus-penetrating nanoparticles for drug and gene delivery to mucosal tissues. Adv. Drug Deliv. Rev. 61, $158-171$.

Lai, S.K., Wang, Y.Y., Hida, K., Cone, R., Hanes, J., 2010. Nanoparticles reveal that human cervicovaginal mucus is riddled with pores larger than viruses. Proc. Natl. Acad. Sci. U.S.A. 107, 598-603.

Lee, J., Grein-Iankovski, A., Narayanan, S., Leheny, R.L., 2017. Nanorod mobility within entangled wormlike micelle solutions. Macromolecules 50, 406-415.

Li, Y.F., Yuan, H.Y., von dem Bussche, A., Creighton, M., Hurt, R.H., Kane, A.B., Gao, H.J., 2013. Graphene microsheets enter cells through spontaneous membrane penetration at edge asperities and corner sites. Proc. Natl. Acad. Sci. U.S.A. 110, 12295-12300.

Masaro, L., Zhu, X.X., 1999. Physical models of diffusion for polymer solutions, gels and solids. Prog. Polym. Sci. 24, 731-775.

McGuckin, M.A., Linden, S.K., Sutton, P., Florin, T.H., 2011. Mucin dynamics and enteric pathogens. Nat. Rev. Microbiol. 9, 265-278.

Ogston, A.G., 1958. The spaces in a uniform random suspension of fibres. Trans. Faraday Soc. 54, $1754-1757$.

Olmsted, S.S., Padgett, J.L., Yudin, A.I., Whaley, K.J., Moench, T.R., Cone, R.A., 2001. Diffusion of macromolecules and virus-like particles in human cervical mucus. Biophys. J. 81, 1930-1937.

Ortega, A., de la Torre, J.G., 2003. Hydrodynamic properties of rodlike and disklike particles in dilute solution. J. Chem. Phys. 119, $9914-9919$.

Peng, Y., Lai, L.P., Tai, Y.S., Zhang, K.C., Xu, X.L., Cheng, X., 2016. Diffusion of ellipsoids in bacterial suspensions. Phys. Rev. Lett. $116,068303$.

Ponchel, G., Irache, J.M., 1998. Specific and non-specific bioadhesive particulate systems for oral delivery to the gastrointestinal tract. Adv. Drug Deliv. Rev. 34, 191-219.

Rodbard, D., Chrambac, A., 1970. Unified theory for gel electrophoresis and gel filtration. Proc. Natl. Acad. Sci. U.S.A. 65, 970-977.

Saxton, M.J., 1994. Anomalous diffusion due to obstacles: a Monte-Carlo study. Biophys. J. 66, 394-401.

Saxton, M.J., 1996. Anomalous diffusion due to binding: a Monte Carlo study. Biophys. J. 70, 1250-1262.

Shi, X.H., von dem Bussche, A., Hurt, R.H., Kane, A.B., Gao, H.J., 2011. Cell entry of one-dimensional nanomaterials occurs by tip recognition and rotation. Nat. Nanotechnol. 6, 714-719.

Skaug, M.J., Mabry, J., Schwartz, D.K., 2013. Intermittent molecular hopping at the solid-liquid interface. Phys. Rev. Lett. 110, 256101.

Smart, J.D., 2005. The basics and underlying mechanisms of mucoadhesion. Adv. Drug Deliv. Rev. 57, 1556-1568.

Stylianopoulos, T., Diop-Frimpong, B., Munn, L.L., Jain, R.K., 2010a. Diffusion anisotropy in collagen gels and tumors: the effect of fiber network orientation. Biophys. J. 99, 3119-3128.

Stylianopoulos, T., Poh, M.Z., Insin, N., Bawendi, M.G., Fukumura, D., Munn, L.L., Jain, R.K., 2010b. Diffusion of particles in the extracellular matrix: The effect of repulsive electrostatic interactions. Biophys. J. 99, 1342-1349.

Tomadakis, M.M., Sotirchos, S.V., 1993. Transport properties of random arrays of freely overlapping cylinders with various orientation distributions. J. Chem. Phys. 98, 616-626.

Vadodaria, S.S., English, R.J., 2016. Aqueous solutions of HEC and hmHEC: effects of molecular mass versus hydrophobic associations on hydrodynamic and thermodynamic parameters. Cellulose 23, 1107-1121.

Viovy, J.L., 2000. Electrophoresis of DNA and other polyelectrolytes: physical mechanisms. Rev. Mod. Phys. 72, 813-872.

Walder, R., Nelson, N., Schwartz, D.K., 2011. Single molecule observations of desorption-mediated diffusion at the solid-liquid interface. Phys. Rev. Lett. 107, 156102.

Wang, J.L., Yao, H.M., Shi, X.H., 2014. Cooperative entry of nanoparticles into the cell. J. Mech. Phys. Solids 73, 151-165

Wang, Y.Y., Lai, S.K., Suk, J.S., Pace, A., Cone, R., Hanes, J., 2008. Addressing the PEG mucoadhesivity paradox to engineer nanoparticles that "slip" through the human mucus barrier. Angew. Chem. Int. Ed. 47, 9726-9729.

Xu, Q.G., Ensign, L.M., Boylan, N.J., Schon, A., Gong, X.Q., Yang, J.C., Lamb, N.W., Cai, S.T., Yu, T., Freire, E., Hanes, J., 2015. Impact of surface polyethylene glycol (PEG) density on biodegradable nanoparticle transport in mucus ex vivo and distribution in vivo. Acs Nano 9, 9217-9227.

Xue, S.L., Li, B., Feng, X.Q., Gao, H.J., 2016. Biochemomechanical poroelastic theory of avascular tumor growth. J. Mech. Phys. Solids 94, 409-432.

Xue, S.L., Li, B., Feng, X.Q., Gao, H.J., 2017. A non-equilibrium thermodynamic model for tumor extracellular matrix with enzymatic degradation. J. Mech. Phys. Solids 104, 32-56.

Yang, K., Ma, Y.Q., 2010. Computer simulation of the translocation of nanoparticles with different shapes across a lipid bilayer. Nat. Nanotechnol. 5, 579-583.

Yasuda, H., Lamaze, C.E., Ikenberr, Ld., 1968. Permeability of solutes through hydrated polymer membranes. Part i. Diffusion of sodium chloride. Makromol. Chem. Macromol. Chem. Phys. 118, 19-35.

Yu, M., Wang, J., Yang, Y., Zhu, C., Su, Q., Guo, S., Sun, J., Gan, Y., Shi, X., Gao, H., 2016. Rotation-facilitated rapid transport of nanorods in mucosal tissues. Nano Lett. 16, 7176-7182.

Zhang, R., Schweizer, K.S., 2016. Statistical mechanical theory of penetrant diffusion in polymer melts and glasses. Macromolecules 49, 5727-5739. 